\title{
On piecewise constant level-set (PCLS) methods for the identification of discontinuous parameters in ill-posed problems
}

\author{
A De Cezaro ${ }^{1}$, A Leitão $^{2}$ and X-C Tai ${ }^{3}$ \\ ${ }^{1}$ Institute of Mathematics Statistics and Physics, Federal University of Rio Grande, \\ Av. Italia km 8, 96201-900 Rio Grande, Brazil \\ 2 Department of Mathematics, Federal University of St. Catarina, PO Box 476, 88040-900 \\ Florianópolis, Brazil \\ ${ }^{3}$ Department of Mathematics, University of Bergen, Johannes Brunsgate 12, N-5008 Bergen, \\ Norway \\ E-mail: decezaromtm@gmail.com,acgleitao@gmail.com and Tai@math.uib.no
}

Received 12 January 2012, in final form 23 August 2012

Published 3 December 2012

Online at stacks.iop.org/IP/29/015003

\begin{abstract}
We investigate level-set-type methods for solving ill-posed problems with discontinuous (piecewise constant) coefficients. The goal is to identify the level sets as well as the level values of an unknown parameter function on a model described by a nonlinear ill-posed operator equation. The PCLS approach is used here to parametrize the solution of a given operator equation in terms of a $L_{2}$ level-set function, i.e. the level-set function itself is assumed to be a piecewise constant function. Two distinct methods are proposed for computing stable solutions of the resulting ill-posed problem: the first is based on Tikhonov regularization, while the second is based on the augmented Lagrangian approach with total variation penalization. Classical regularization results (Engl H W et al 1996 Mathematics and its Applications (Dordrecht: Kluwer)) are derived for the Tikhonov method. On the other hand, for the augmented Lagrangian method, we succeed in proving the existence of (generalized) Lagrangian multipliers in the sense of (Rockafellar R T and Wets R J-B 1998 Grundlehren der Mathematischen Wissenschaften (Berlin: Springer)). Numerical experiments are performed for a 2D inverse potential problem (Hettlich F and Rundell W 1996 Inverse Problems 12 251-66), demonstrating the capabilities of both methods for solving this ill-posed problem in a stable way (complicated inclusions are recovered without any a priori geometrical information on the unknown parameter).
\end{abstract}

(Some figures may appear in colour only in the online journal) 


\section{Introduction}

\subsection{Ill-posed problems and adequate parameter spaces}

Several ill-posed inverse problems of interest consist of identifying an unknown physical quantity, $u \in X$, that can be represented by a piecewise constant real function over a bounded given domain $\Omega$, from the set of data $y \in Y$, where $X, Y$ are Hilbert spaces. The relation between the unknown parameter function and the problem data is typically described by the (possibly nonlinear) model

$$
F(u)=y,
$$

where $F: D(F) \subset X \rightarrow Y$; meaning that the set of data is obtained by indirect measurements of the parameter. In practical applications, the exact data $y \in Y$ are, in general, not known. One is given only approximate measured data $y^{\delta} \in Y$, corrupted by noise of level $\delta>0$, satisfying

$$
\left\|y^{\delta}-y\right\|_{Y} \leqslant \delta
$$

In the case where the unknown function $u$ is piecewise constant distinguishing between two given values, level-set approaches were considered in [36, 27, 20, 6-9, 14, 28]. In this case, since the level values of $u$ are known, one needs only to identify the level sets of $u$, and the problem in (1) reduces to a shape identification problem. In the case where the unknown function $u$ is a piecewise constant function distinguishing between several given values, multiple level-set approaches were considered in $[8,10,13]$.

If the level values of $u$ are also unknown, then the inverse problem becomes harder, since one has to identify both the level sets and the level values of the unknown parameter $u$. In this case, the dimension of the parameter space increases by the number of unknown level values $[39,40]$.

The methods discussed in this paper are designed to solve the operator equation (1) under the assumption that the parameter function $u$ is a piecewise constant function taking only two possible (unknown) values, i.e. $u(x) \in\left\{c^{1}, c^{2}\right\}$ a.e. in $\Omega \subset \mathbb{R}^{d}$. In other words, one can assume the existence of an open measurable set $D \subset \subset \Omega$ s.t.

$$
u(x)=c^{1}, x \in D=: D_{1} \text { and } u(x)=c^{2}, x \in \Omega / D=: D_{2} .
$$

The list of relevant applications matching this framework is large (see, e.g., [39, 41, 14, $38,9,7]$ and the references therein). It is worth mentioning that the methods presented here can be extended in a straightforward way to the case where the unknown parameter takes any finite number of values $[32,13,37]$.

\subsection{PCLS framework: reparametrizing the parameter space}

In this paper, a piecewise constant level-set (PCLS) approach [32, 44] is used to represent the unknown parameter in (1) of the form (3), i.e. the $L_{\infty}$-function $u$ can be represented using a smooth operator $P_{p c}: L_{2}(\Omega) \rightarrow L_{2}(\Omega)$ and a discontinuous (piecewise constant) level-set function $\phi \in L_{2}(\Omega)$. Here we assume that $\phi(x)=i-1, x \in D_{i}$, with $D_{i}$ defined as in (3). Note that, using the auxiliary functions $\psi_{1}(t):=1-t$ and $\psi_{2}(t):=t$, the characteristic functions of the subdomains $D_{i}$ can be written in the form $\chi_{D_{i}}(x)=\psi_{i}(\phi(x)), i=1,2$. Therefore, a solution $u$ of (1) can be parametrized by the operator

$$
u=c^{1} \psi_{1}(\phi)+c^{2} \psi_{2}(\phi)=: P_{p c}\left(\phi, c^{j}\right) .
$$

The piecewise constant assumption on $\phi$ corresponds to the constraint $\mathcal{K}(\phi)=0$, where $\mathcal{K}(\phi):=(\phi)(\phi-1)$ is a smooth nonlinear operator. The constraint described by $\mathcal{K}(\phi)=0$ 
can alternatively be expressed in the form $K(\phi)=0$, where $K(\phi)=\sqrt{|\phi||\phi-1|}$. Note that, differently from $\mathcal{K}(\cdot)$, the operator $K(\cdot)$ is not differentiable.

An alternative way (commonly found in the literature) to represent the piecewise constant assumption on $\phi$ is given by the double well potentials $(\phi)^{2 n}(\phi-1)^{2 n}=0, n \in \mathbb{N}$ [44]. Here, we use a low-order polynomial to represent this constraint. The advantage of this choice resides in the fact that the corresponding operator $\mathcal{K}$ is continuous from $L_{2}(\Omega)$ to $L_{1}(\Omega)$. On the other hand, the operator $K$ is continuous from $L_{2}(\Omega)$ to $L_{2}(\Omega)$. Such regularity properties (which are necessary for the analysis derived in section 2 ) are not shared by the double well potentials above.

Within this framework, the inverse problem in (1), with data given as in (2), and solution of the form (3), can be written in the form of the abstract operator equation

$$
F\left(P_{p c}\left(\phi, c^{j}\right)\right)=y^{\delta}, \quad \text { where } \phi \in\left\{L_{2}(\Omega) ; \mathcal{K}(\phi)=0\right\} \text { and } c^{j} \in \mathbb{R} \text {. }
$$

Remark 1 (A few words on the PCLS framework). Continuous level-set functions. Iterative methods based on this framework produce a sequence of level-set functions which are typically continuous. Only at the end of the iterative process, a PCLS (i.e. a function satisfying the constraint $\mathcal{K}(\phi)=0$ ) is obtained [38].

Relation to phase field methods. Level-set methods based on the PCLS framework belong to the family of binary level-set methods [32,44], which are similar to the well-known phase field methods $[2,1,4]$ used for phase transition type problems.

PCLS approach versus standard level-set approach. The standard level-set approach $[36,27]$ for problem (1) consists in introducing a smooth level-set function $\phi\left(\right.$ e.g., $\phi \in H^{1}(\Omega)$ ) which acts as a regularization of the parameter space. The discontinuities of the parameter $u$ are represented implicitly by the zero level-set of $\phi$. In this approach, the Heaviside projector $H$ is used to represent a solution of (1) in the form $u=c^{2} H(\phi)+c^{1}(1-H(\phi))=: P_{l s}\left(\phi, c^{j}\right)$.

Here $u(x)=c^{i}, x \in D_{i}$, where the sets $D_{i}$ correspond to $D_{1}=\{x \in \Omega ; \phi(x) \geqslant 0\}$ and $D_{2}=\{x \in \Omega ; \phi(x)<0\}$. Thus, the operator $P_{l s}$ establishes a straightforward relation between the level sets of $\phi$ and the sets $D_{i}$, representing the a priori knowledge about the solution $u$.

Within this framework, the inverse problem in (1), with data given as in (2), can be written in the form of the operator equation $F\left(P_{s}\left(\phi, c^{j}\right)\right)=y^{\delta}$. In order to obtain approximate solutions to this equation, different regularization schemes have been proposed in the literature so far, e.g., Tikhonov regularization using $T V[7,8,37]$, or Tikhonov regularization using $T V-H^{1}$ $[20,13]$.

\subsection{Main contributions}

The main contribution of this paper is to address two reconstruction methods for solving equation (5) in a stable way. The first one consists essentially in applying Tikhonov regularization with total variation penalization [20] to the system: $\left[F\left(P_{p c}\left(\phi, c^{j}\right)\right), \mathcal{K}(\phi)\right]=$ $\left[y^{\delta}, 0\right]$.

The second method is based on the augmented Lagrangian approach [3, 25] for a (regularized) constrained optimization problem corresponding to the operator equation (5). From the numerical point of view, augmented Lagrangian methods have been intensively investigated in the imaging community in connection to binary reconstruction problems $[32,26,44]$. From the analytical point of view, these methods were considered in $[18,19,42]$ 
1.3.1. First reconstruction method: Tikhonov regularization. Approximate solutions to (5) can be obtained by minimizing the Tikhonov functional

$$
\mathcal{G}_{\alpha}\left(\phi, c^{j}\right):=\left\|F\left(P_{p c}\left(\phi, c^{j}\right)\right)-y^{\delta}\right\|_{Y}^{2}+\mu\|\mathcal{K}(\phi)\|_{L_{1}}+\alpha\left\{\left|P_{p c}\left(\phi, c^{j}\right)\right|_{\mathrm{BV}}+\left\|c^{j}\right\|_{\mathbb{R}^{2}}^{2}\right\} .
$$

Note that the minimization of the functional $\mathcal{G}_{\alpha}$ furnishes a regularized solution to the system of operator equations:

$$
\left[\begin{array}{c}
F\left(P_{p c}\left(\phi, c^{j}\right)\right) \\
\mathcal{K}(\phi)
\end{array}\right]=\left[\begin{array}{l}
y^{\delta} \\
0
\end{array}\right]
$$

The penalization term in (6) corresponds essentially to $T V$ regularization, while $\mu>0$ plays the role of a scaling factor balancing the misfits of the two operator equations. (The correct choice of the constant $\mu$ is very important in practical applications, since the first misfit term depends crucially on the scale of the data, while the second does not.)

It is worth noting that, in the limit case $\alpha \rightarrow 0,{ }^{4}$ the minimizers $\left(\phi_{\alpha}, c_{\alpha}^{j}\right)$ of $\mathcal{G}_{\alpha}$ converge to some limit $\left(\bar{\phi}, \bar{c}^{j}\right)$ satisfying $F\left(P_{p c}(\bar{\phi}, \bar{c})\right)=y$ and $\mathcal{K}(\bar{\phi})=0$ (see theorem 4 below). Thus, the limit level-set function $\bar{\phi}$ is indeed piecewise constant (as suggested by the acronym PCLS).

Here, we extend the Tikhonov regularization approach in [13] to the functional $\mathcal{G}_{\alpha}$ in (6), and prove convergence and stability results for the proposed variational approach.

In [12], a similar Tikhonov functional was investigated (numerically) in connection with the PCLS framework, and a corresponding iterative method was derived. Here we are able to improve the efficiency of that iterative method by appropriately choosing the parameter $\mu$. Namely, instead of choosing $\mu$ constant along the iterations, we propose starting the iteration with a small value $\mu_{0}$ and slowly increase it with the iteration. This allowed us to reduce the numerical effort to the half (see numerical experiments in section 5.2).

1.3.2. Second reconstruction method: augmented Lagrangian. Approximate solutions to (5) can be obtained by applying the augmented Lagrangian method to the regularized constrained optimization problem

$$
\left\{\begin{array}{l}
\min \mathcal{F}_{\alpha}\left(\phi, c^{j}\right):=\left\|F\left(P_{p c}\left(\phi, c^{j}\right)\right)-y^{\delta}\right\|^{2}+\alpha\left\{\left|P_{p c}\left(\phi, c^{j}\right)\right|_{\mathrm{BV}}+\left\|c^{j}\right\|_{\mathbb{R}^{2}}^{2}\right\}, \\
\text { s.t. } \phi \in\left\{L_{2}(\Omega) ; K(\phi)=0\right\} .
\end{array}\right.
$$

Due to lack of convexity of the constraint $K(\phi)=0$ and the functional $\mathcal{F}_{\alpha}$, classical Lagrange methods $[34,3]$ cannot be applied in a straightforward way to solve the constrained optimization problem (8). Alternatively, we introduce the (augmented) Lagrangian functional $\mathcal{G}_{L, \alpha}$, which is formally defined by

$$
\begin{gathered}
\mathcal{G}_{L, \alpha}\left(\phi, c^{j} ; \lambda, \mu\right):=\left\|F\left(P_{p c}\left(\phi, c^{j}\right)\right)-y^{\delta}\right\|_{Y}^{2}+\mu\|K(\phi)\|_{L_{2}}+\int_{\Omega} \lambda K(\phi) \\
+\alpha\left\{\left|P_{p c}\left(\phi, c^{j}\right)\right|_{\mathrm{BV}}+\left\|c^{j}\right\|_{\mathbb{R}^{2}}^{2}\right\},
\end{gathered}
$$

and search for 'generalized' multipliers $(\lambda, \mu)$ in the sense of [35, chapter $\left.11 . \mathrm{K}^{*}\right]$ (see also [30, chapter 5]). The scalar $\mu>0$ in (9) is a penalty factor that allows one to establish a duality relation for problems of non-convex type, while the $L^{2}(\Omega)$-vector $\lambda$ can be interpreted as a 'generalized' Lagrange multiplier.

According to the abstract augmented Lagrangian framework followed here, one aims to find a vector $\bar{\lambda}$ supporting an exact penalty representation for the primal problem, as well as a corresponding penalty factor $\bar{\mu}$ (see definition 3 in section 3 ). Once such a pair $(\bar{\lambda}, \bar{\mu})$ is

4 Recall that in the presence of noise, $\delta>0$, the regularization parameter $\alpha$ is a function of the noisy level, i.e. $\alpha=\alpha(\delta)$; see theorem 4 . 
known, an approximate solution to (7) can be found by solving an unconstrained optimization problem (just as in the classical Lagrange theory).

The augmented Lagrangian approach followed here can be seen as a combination of the penalty function method and the Lagrangian multiplier method and is able to eliminate many disadvantages associated with either method alone [3]. In comparison with the quadratic penalty method for constrained optimization problems, the convergence of the augmented Lagrangian method usually does not require the penalty parameter to tend to infinity [3]. This result eliminates (or at least moderates) the ill-conditioning in the penalty method.

Another advantage of the augmented Lagrangian method is that its convergence rate is considerably better than that of the penalty method (see [35, 3] and section 4). It is worth noting that the existence of an exact penalty function for the particular primal problem (8) is essential for the successful analysis of this approach.

The penalization term in (9) has the same meaning as the one in (6), and $\alpha>0$ plays the role of a regularization parameter.

\subsection{Outline of the paper}

In section 2, we present a convergence analysis for the Tikhonov method (6) based on the PCLS approach. A corresponding algorithm is derived (see subsection 2.1). In section 3, we present a convergence analysis for the augmented Lagrange method (9) based on the PCLS approach. A corresponding algorithm is presented in subsection 3.1. Section 4 is devoted to the investigation of numerical methods. The benchmark two-dimensional inverse potential problem is introduced [20, 13]. A numerical algorithm (of PCLS type) based on the Tikhonov method is derived and discussed for solving this inverse problem. A second algorithm (of PCLS type) based on the augmented Lagrangian method is derived and implemented for the same inverse problem. In section 5, numerical tests with the proposed algorithms are presented. Experiments with exact and noisy data are both considered, and the performances of the two reconstruction methods proposed in this manuscript are compared.

\section{PCLS approach and the Tikhonov method}

We start this section briefly recalling some results related to the convergence analysis of the regularization method based on the PCLS approach.

We shall consider the model problem described as in the introduction under the following general assumptions.

(A1) $\Omega \subseteq \mathbb{R}^{d}, d=2$, is bounded with piecewise $C^{1}$ boundary $\partial \Omega$.

(A2) The operator $F: \mathbb{D} \subset L_{p}(\Omega) \rightarrow Y$ is continuous and Fréchet-differentiable on $\mathbb{D}$ with respect to the $L_{p}$-topology, where $1 \leqslant p<d /(d-1)=2$.

(A3) $\alpha$ and $\mu$ denote positive parameters.

(A4) There exists $\hat{u} \in L_{\infty}(\Omega)$ satisfying $F(\hat{u})=y$. Moreover, there exists a function $\hat{\phi} \in \operatorname{BV}(\Omega) \subset L_{2}(\Omega)$ and constants $\hat{c}^{1} \neq \hat{c}^{2} \in \mathbb{R}$ such that $P_{p c}\left(\hat{\phi}, \hat{c}^{j}\right)=\hat{u}$ and $\mathcal{K}(\hat{\phi})=0$.

Let $\tilde{D} \subset \Omega$ be a open and bounded subset, with the Lebesgue measure $|\tilde{D}|>\gamma>0$ for a fix $\gamma$. We define the following subset of BV:

$$
\mathrm{BV}_{0}(\Omega):=\{\phi \in \mathrm{BV}(\Omega): \phi(x)=0, \quad \text { a.e. } x \in \tilde{D}\} .
$$

A straightforward consequence of the definition of $P_{p c}(\cdot, \cdot)$ is that, for fixed constants $c^{j}$, this operator is $1-1$, continuous and continuously differentiable from $L_{2}(\Omega)$ onto $L_{2}(\Omega)$. Consequently, the set of admissible vectors for the Tikhonov functional in (6) is defined in the following way. 
Definition 1. Let the operator $P_{p c}$ defined as in (4) and $\tau>0$. A vector $\left(\phi, c^{j}\right) \in L^{2}(\Omega) \times \mathbb{R}^{2}$ is called admissible when $\phi \in \mathrm{BV}_{0}(\Omega)$ and $\left|c^{2}-c^{1}\right| \geqslant \tau$.

From (4), it follows that $P_{p c}$ maps admissible vectors to $\operatorname{BV}(\Omega)$. Next we briefly recall some basic facts about the space $\operatorname{BV}(\Omega)$. For a proof, we refer the reader to [17, chapter 5].

Lemma 1. The following assertions hold true.

(i) The semi-norm $|\cdot|_{\mathrm{BV}}$ is weakly lower semi-continuous with respect to $L_{p}$-convergence, i.e. if $\left\{x_{k}\right\} \in \mathrm{BV}(\Omega)$ converges to $x$ in the $L_{p}$-norm, then $x \in \operatorname{BV}(\Omega)$ and $|x|_{\mathrm{BV}} \leqslant$ $\liminf \operatorname{in}_{k \rightarrow \infty}\left|x_{k}\right|_{\mathrm{BV}}$.

(ii) $\mathrm{BV}(\Omega)$ is compactly embedded in $L_{p}(\Omega)$ for $1 \leqslant p<d /(d-1)$. Consequently, any bounded sequence $\left\{x_{k}\right\} \in \mathrm{BV}(\Omega)$ has a subsequence converging in $L_{p}(\Omega)$ to some $x \in \operatorname{BV}(\Omega)$.

The following lemma is devoted to the investigation of relevant properties of operators $\mathcal{K}$ and $P_{p c}$, respectively.

Lemma 2. Let $\mathcal{K}, K$ be the operators defined in section 1 and $P_{p c}$ the operator defined in (4). For $1 \leqslant p<2$, the following assertions hold true.

(i) $\mathcal{K}$ and K are continuous maps from $L_{2}(\Omega)$ to $L_{1}(\Omega)$ and from $L_{2}(\Omega)$ to $L_{2}(\Omega)$ respectively; additionally the functional $\|\mathcal{K}(\cdot)\|_{L_{1}}$, defined in $L_{2}(\Omega)$, is weakly lower semi-continuous.

(ii) If $\|\mathcal{K}(\phi)\|_{L_{1}}=0$ or $\|K(\phi)\|_{L_{2}}=0$ for some $\phi \in L_{2}(\Omega)$, then $\phi(x) \in\{0,1\}$ a.e. in $\Omega$.

(iii) For every admissible vector $\left(\phi, c^{j}\right)$ we have $\left|P_{p c}\left(\phi, c^{j}\right)\right|_{\mathrm{BV}} \geqslant \tau|\phi|_{\mathrm{BV}}$.

(iv) $\mathrm{BV}_{0}(\Omega)$ is a closed subset of $\mathrm{BV}(\Omega)$ with respect to the $L_{p}(\Omega)$ convergence. In other words, if $\phi_{k} \in \mathrm{BV}_{0}(\Omega)$ is a sequence converging to $\phi \in \mathrm{BV}(\Omega)$ with respect to the $L_{p}(\Omega)$-topology, then $\phi \in \mathrm{BV}_{0}(\Omega)$.

(v) For every admissible vector $\left(\phi, c^{j}\right)$, there exist a constant $c>0$ such that $\left|P_{p c}\left(\phi, c^{j}\right)\right|_{\mathrm{Bv}} \geqslant$ $c\|\phi\|_{L_{2}(\Omega)}$.

Moreover, if $\left(\phi_{k}, c_{k}^{j}\right)$ is a sequence of admissible vectors converging in $L_{p}(\Omega) \times \mathbb{R}^{2}$ to some admissible vector $\left(\phi, c^{j}\right)$, then

(vi) $P_{p c}\left(\phi_{k}, c_{k}^{j}\right)$ converges to $P_{p c}\left(\phi, c^{j}\right)$ in $L_{p}(\Omega)$.

(vii) $\left|P_{p c}\left(\phi, c^{j}\right)\right|_{\mathrm{BV}} \leqslant \liminf _{k \rightarrow \infty}\left|P_{p c}\left(\phi_{k}, c_{k}^{j}\right)\right|_{\mathrm{BV}}$.

Proof. The continuity of $\mathcal{K}$ follows from $\int_{\Omega}|\mathcal{K}(\phi)-\mathcal{K}(\psi)| \leqslant \int_{\Omega}|\phi||\phi-\psi|+\int_{\Omega} \mid \psi-$ 1||$\psi-\phi \mid$, together with the Cauchy-Schwarz inequality. The continuity of $K$ follows from a similar argumentation (additionally, one has to apply the inequality $2 a b \leqslant a^{2}+b^{2}$ ).

To verify the last assertion of item (i), note that equation $\mathcal{K}(\phi)=0$ in (7) is equivalent to $\tilde{\mathcal{K}}(\phi)=1 / 4$, where $\tilde{\mathcal{K}}(\phi):=\mathcal{K}(\phi)+1 / 4$. Thus, it is enough to prove that the functional $\|\tilde{\mathcal{K}}(\cdot)\|_{L_{1}}$ is weakly l.s.c. Since the real function $t \mapsto|\tilde{\mathcal{K}}(t)|$ is convex, this property follows from Dacorogna [11, theorem 1.1, p 7; and subsequent remark, p 8]. Assertion (ii) follows immediately form the definition of $\mathcal{K}$ and $K$.

Assertion (iii) follows form [12, lemma 9 (i)]. Minkowski inequality imply

$$
\left(\int_{\tilde{D}}|\phi|^{p} \mathrm{~d} x\right)^{\frac{1}{p}} \leqslant\left(\int_{\tilde{D}}\left|\phi-\phi_{k}\right|^{p} \mathrm{~d} x\right)^{\frac{1}{p}}+\left(\int_{\tilde{D}}\left|\phi_{k}\right|^{p} \mathrm{~d} x\right)^{\frac{1}{p}} \leqslant\left\|\phi-\phi_{k}\right\|_{L_{p}(\Omega)},
$$

and (iv) is proved. Assertion (v) follows from assertion (iii) and the Poincaré inequality for BV functions [17, theorem 1, item (ii), p 189]. Finally, items (vi) and (vii) follows from [12, lemma 9, items (ii) and (iii)], respectively. 
Note that lemma $2(\mathrm{v})$ guarantees the coercivity of the functional $\left|P_{p c}(\cdot, \cdot)\right|_{\mathrm{BV}}$ (w.r.t. the $L_{2}$-norm) on the set of admissible parameters. This lemma contains the essential tools needed to derive the main convergence analysis results for the PCLS approach. Let $R_{p c}\left(\phi, c^{j}\right):=\left|P_{p c}\left(\phi, c^{j}\right)\right|_{\mathrm{BV}}+\left\|c^{j}\right\|_{\mathbb{R}^{2}}^{2}$ be the penalization term of $\mathcal{G}_{\alpha}$ in (6). Given $\alpha, \mu>0$, the next result guarantees the well posedness of functional $\mathcal{G}_{\alpha}$.

Theorem 3. The functional $\mathcal{G}_{\alpha}$ in (6) attains minimizers on the set of admissible vectors.

Proof. Let $\left\{\left(\phi_{k}, c_{k}^{j}\right)\right\}$ be a minimizing sequence for $\mathcal{G}_{\alpha}$, i.e. a sequence of admissible vectors satisfying $\mathcal{G}_{\alpha}\left(\phi_{k}, c_{k}^{j}\right) \rightarrow \inf \mathcal{G}_{\alpha}, k \rightarrow \infty$. Then, $\left\{R_{p c}\left(\phi_{k}, c_{k}^{j}\right)\right\}$ is a bounded sequence of real numbers and it follows from lemma $2(\mathrm{v})$ the existence of a subsequence $\left\{\phi_{k}\right\}$ and $\bar{\phi} \in L_{2}(\Omega)$ such that $\phi_{k} \rightarrow \bar{\phi}$ in $L_{2}(\Omega)$. Moreover, from lemma 2 (iii) and lemma 1 (ii) we conclude that $\bar{\phi} \in \mathrm{BV}(\Omega)$ and that this subsequence also satisfies $\phi_{k} \rightarrow \bar{\phi}$ in $L_{p}(\Omega)$. Therefore, from lemma 2 (iv), $\bar{\phi} \in \mathrm{BV}_{0}$.

On the other hand, the boundedness of $\left\{R_{p c}\left(\phi_{k}, c_{k}^{j}\right)\right\}$ also guarantees the existence of subsequences $\left\{c_{k}^{j}\right\}$ converging to $\bar{c}^{j}$ in $\mathbb{R}^{2}$.

Clearly $\left(\bar{\phi}, \bar{c}^{j}\right)$ is an admissible vector. Moreover, from (A2), lemma 2 (i), (vi) and (vii) it follows that

$$
\begin{aligned}
\inf \mathcal{G}_{\alpha} & =\lim _{k \rightarrow \infty} \mathcal{G}_{\alpha}\left(\phi_{k}, c_{k}^{j}\right) \\
& =\liminf _{k \rightarrow \infty}\left\{\left\|F\left(P_{p c}\left(\phi_{k}, c_{k}^{j}\right)\right)-y^{\delta}\right\|_{Y}^{2}+\mu\left\|\mathcal{K}\left(\phi_{k}\right)\right\|_{L_{1}}+\alpha R_{p c}\left(\phi_{k}, c_{k}^{j}\right)\right\} \\
& \geqslant\left\|F\left(P_{p c}\left(\bar{\phi}, \bar{c}^{j}\right)\right)-y^{\delta}\right\|_{Y}^{2}+\mu\|\mathcal{K}(\bar{\phi})\|_{L_{1}}+\alpha R_{p c}\left(\bar{\phi}, \bar{c}^{j}\right)=\mathcal{G}_{\alpha}\left(\bar{\phi}, \bar{c}^{j}\right),
\end{aligned}
$$

proving that $\left(\bar{\phi}, \bar{c}^{j}\right)$ minimizes $\mathcal{G}_{\alpha}$.

Standard convergence and stability results (cf [12, theorem 7] or [13, theorems 8 and 9]) hold true for the Tikhonov method based on the PCLS approach. The proof uses classical techniques from the analysis of Tikhonov-type regularization methods [16] and thus is omitted.

Theorem 4. Assume that we have exact data, i.e. $\delta=0$ in (2), and $\mu>0$. For every $\alpha>0$ denote by $\left(\phi_{\alpha}, c_{\alpha}^{j}\right)$ a minimizer of $\mathcal{G}_{\alpha}$ on the set of admissible vectors. Then, for every sequence of positive numbers $\left\{\alpha_{k}\right\}$ converging to zero there exists a subsequence such that $\left(\phi_{\alpha_{k}}, c_{\alpha_{k}}^{j}\right)$ is strongly convergent in $L_{p}(\Omega) \times \mathbb{R}^{2}$. Moreover, the limit is a solution of (7) with $y^{\delta}=y$.

In the case of noisy data, let $\alpha=\alpha(\delta)$ be a positive function with $\lim _{\delta \rightarrow 0} \alpha(\delta)=0$ and $\lim _{\delta \rightarrow 0} \delta^{2} / \alpha(\delta)=0$. Given a sequence $\left\{\delta_{k}\right\}$ of positive numbers converging to zero and $\left\{y^{\delta_{k}}\right\} \in Y$ be corresponding noisy data satisfying (2), there exist a subsequence, denoted again by $\left\{\delta_{k}\right\}$, and a sequence $\left\{\alpha_{k}:=\alpha\left(\delta_{k}\right)\right\}$ such that $\left(\phi_{\alpha_{k}}, c_{\alpha_{k}}^{j}\right)$ converges in $L_{p}(\Omega) \times \mathbb{R}^{2}$ to a solution of (7) with $y^{\delta}=y$.

Note that the limit elements $\left(\phi, c^{j}\right)$ obtained from the convergence-stability theorem 4 satisfy not only $F\left(P_{p c}\left(\phi, c^{j}\right)\right)=y$, but also $\|\mathcal{K}(\phi)\|_{L_{1}}=0$. Therefore, due to lemma 2 (ii), we conclude that the limit level-set function $\phi$ is piecewise constant.

\subsection{An iterative algorithm based on the Tikhonov method}

The iterative algorithm based on the regularization method for PCLS approach proposed in this paper is an explicit iterative method based on the operator splitting technique [21, 29] and derived from the optimality conditions for the Tikhonov functional $\mathcal{G}_{\alpha}$ in (6). First the operator $\mathcal{G}_{\alpha}$ is split in the sum $\mathcal{G}_{\alpha}\left(\phi, c^{j}\right)=\mathcal{G}_{\alpha}^{1}\left(\phi, c^{j}\right)+\mathcal{G}_{\alpha}^{2}(\phi)$, where

$$
\begin{aligned}
\mathcal{G}_{\alpha}^{1}\left(\phi, c^{j}\right) & :=\left\|F\left(P_{p c}\left(\phi, c^{j}\right)\right)-y^{\delta}\right\|_{Y}^{2}+\alpha\left\{\left|P_{p c}\left(\phi, c^{j}\right)\right|_{\mathrm{BV}}+\left\|c^{j}\right\|_{\mathbb{R}^{2}}^{2}\right\} \\
\mathcal{G}_{\alpha}^{2}(\phi) & :=\mu\|\mathcal{K}(\phi)\|_{L_{1}(\Omega)} .
\end{aligned}
$$


Each step of the iterative method consists of two parts.

(i) The iterate $\left(\phi_{k}, c_{k}^{j}\right)$ is updated using an explicit gradient step w.r.t. the operator $\mathcal{G}_{\alpha}^{1}$, i.e.

$$
\phi_{k+1 / 2}:=\phi_{k}-\frac{\partial}{\partial \phi} \mathcal{G}_{\alpha}^{1}\left(\phi_{k}, c_{k}^{j}\right), \quad c_{k+1 / 2}^{j}:=c_{k}^{j}-\frac{\partial}{\partial c^{j}} \mathcal{G}_{\alpha}^{1}\left(\phi_{k}, c_{k}^{j}\right),
$$

where

$$
\begin{aligned}
\frac{\partial}{\partial \phi} \mathcal{G}_{\alpha}^{1}\left(\phi, c^{j}\right)= & \left(c^{2}-c^{1}\right) F^{\prime}\left(P_{p c}\left(\phi, c^{j}\right)\right)^{*}\left[F\left(P_{p c}\left(\phi, c^{j}\right)\right)-y^{\delta}\right] \\
& -\frac{\alpha}{2}\left(c^{2}-c^{1}\right) \nabla \cdot\left[\nabla P_{p c}(\phi) /\left|\nabla P_{p c}(\phi)\right|\right], \\
\frac{\partial}{\partial c^{1}} \mathcal{G}_{\alpha}^{1}\left(\phi, c^{j}\right)= & {\left[F^{\prime}\left(P_{p c}\left(\phi, c^{j}\right)\right)(1-\phi)\right]^{*}\left(F\left(P_{p c}\left(\phi, c^{j}\right)\right)-y^{\delta}\right), } \\
& -\frac{\alpha}{2}(1-\phi) \nabla \cdot\left[\nabla P_{p c}(\phi) /\left|\nabla P_{p c}(\phi)\right|\right]+c^{1}, \\
\frac{\partial}{\partial c^{2}} \mathcal{G}_{\alpha}^{1}\left(\phi, c^{j}\right)= & {\left[F^{\prime}\left(P_{p c}\left(\phi, c^{j}\right)\right)(\phi)\right]^{*}\left(F\left(P_{p c}\left(\phi, c^{j}\right)\right)-y^{\delta}\right) } \\
& -\frac{\alpha}{2}(\phi) \nabla \cdot\left[\nabla P_{p c}(\phi) /\left|\nabla P_{p c}(\phi)\right|\right]+c^{2} .
\end{aligned}
$$

(ii) The obtained approximation $\left(\phi_{k+1 / 2}, c_{k+1 / 2}^{j}\right)$ is improved by giving a gradient step w.r.t. the operator $\mathcal{G}_{\alpha}^{2}$, i.e.

$$
\phi_{k+1}:=\phi_{k+1 / 2}-\frac{d}{d \phi} \mathcal{G}_{\alpha}^{2}\left(\phi_{k+1 / 2}\right), \quad c_{k+1}^{j}:=c_{k+1 / 2}^{j},
$$

where

$$
\frac{\partial}{\partial \phi} \mathcal{G}_{\alpha}^{2}(\phi)=\mu(2 \phi-3) \mathcal{K}(\phi) /|\mathcal{K}(\phi)|,
$$

In [38], a similar operator splitting strategy was used to minimize a Tikhonov functional related to an elliptic inverse problem in EIT. For a comprehensive study of operator splitting methods we refer the reader to Glowinski's book [21].

In the numerical implementation of the algorithm described above, the issue of the choice of $\mu$ is critical.

If a large value of $\mu$ is chosen, then the iterates $\phi_{k}$ satisfy the constraint $\mathcal{K}\left(\phi_{k}\right)=0$ (i.e. become piecewise constant) after a few steps and the iteration stagnates. However, the corresponding approximate solution $P_{p c}\left(\phi_{k}, c_{k}^{j}\right)$ is far from the true parameter.

Typically, a very small constant value $\mu>0$ has to be chosen. This allows the computation of a much precise approximation for the true parameter. However, this choice of $\mu$ leads to a very slow convergence of the operator splitting scheme, since a large number of iterative steps is required in order to enforce the constraint $\mathcal{K}\left(\phi_{k}\right)=0$.

This facts suggested the use of a slight variation of the iteration described above. Instead of choosing a constant value for $\mu$, we start the algorithm above using a small value $\mu=\mu_{0}$ (i.e. the operator splitting scheme consists basically of it's first part). During the iteration, the value of $\mu$ increases gradually according to a pre-defined strategy [38]. This alternative implementation of the operator splitting scheme leads to much faster and stable algorithm, as discussed in section 5 .

Remark 2. The PCLS approach described above is characterized by a constraint enforcing either $\phi=0$ or $\phi=1$ in $\Omega$. It is worth noting that the resulting (two steps) level-set algorithm relates to the phase field method used by the dynamic interface community to analyze front propagation problems $[2,5]$. 


\section{PCLS approach and augmented Lagrangian}

This section is devoted to the analysis of the augmented Lagrangian approach introduced in section 1. In what follows, we prove two main results: (i) existence of zero duality gap ${ }^{5}$; (ii) exact penalty representation for the duality scheme induced by the augmented Lagrangian function (9).

The main tool in our analysis is abstract convexity, which recently became a natural language to investigate duality-schemes via augmented Lagrangian-type functions [35].

For the rest of this section, we adopt the notation:

- $\Gamma$ is the set valued function satisfying $\Gamma(z):=\left\{\phi \in L_{2}(\Omega) ; K(\phi)=z\right\}, z \in L_{2}(\Omega)$.

- The set distance function in $L_{2}(\Omega)$ is denoted here by $\delta$ (recalling, this is the function defined by $\delta_{A}(z):=0$, if $z \in A$ and $\delta_{A}(z):=+\infty$, otherwise).

In the following, we introduce some functions that are necessary for the forthcoming analysis:

- $\widetilde{\mathcal{F}}_{\alpha}\left(\phi, c^{j}\right)=\mathcal{F}_{\alpha}\left(\phi, c^{j}\right)$, if $\phi \in \Gamma(0)$ and $\widetilde{\mathcal{F}}_{\alpha}\left(\phi, c^{j}\right)=+\infty$, otherwise.

- A dualizing parametrization function [35] for $\widetilde{\mathcal{F}}_{\alpha}$ is chosen in the following way: $f: L_{2}(\Omega) \times \mathbb{R}^{2} \times L_{2}(\Omega) \rightarrow \mathbb{R}, f\left(\phi, c^{j}, z\right):=\mathcal{F}_{\alpha}\left(\phi, c^{j}\right)+\delta_{\Gamma(z)}(\phi)$. The function $f$ satisfies the property $f\left(\phi, c^{j}, 0\right)=\widetilde{\mathcal{F}}_{\alpha}\left(\phi, c^{j}\right),\left(\phi, c^{j}\right) \in L_{2}(\Omega) \times \mathbb{R}^{2}$.

- The perturbation function (of the primal problem) related to this duality parametrization, is given by $\theta: L_{2}(\Omega) \rightarrow \mathbb{R}, \theta(z):=\inf _{\left(\phi, c^{j}\right) \in L_{2}(\Omega) \times \mathbb{R}^{2}} f\left(\phi, c^{j}, z\right)$. Note that assumption (A4) guarantees $\theta(0)<+\infty$.

- A coupling function $\rho: L_{2}(\Omega) \times L_{2}(\Omega) \times \mathbb{R}_{+} \rightarrow \mathbb{R}$ is defined by $\rho(z, \lambda, \mu):=$ $-\langle\lambda, z\rangle-\mu\|z\|_{L_{2}}$, where $\langle\cdot, \cdot\rangle$ denotes the usual $L_{2}$-inner product.

- The augmented Lagrangian functional induced by the coupling function $\rho$ reads

$$
\mathcal{G}_{L, \alpha}\left(\phi, c^{j} ; \lambda, \mu\right)=\inf _{z \in L_{2}(\Omega)}\left\{f\left(\phi, c^{j}, z\right)-\rho(z, \lambda, \mu)\right\} .
$$

It is straightforward to verify that, with the above definitions, $\mathcal{G}_{L, \alpha}\left(\phi, c^{j} ; \lambda, \mu\right)$ coincides with the functional introduced in (9). Moreover, $\mathcal{G}_{L, \alpha}\left(\phi, c^{j} ; \lambda, \mu\right)$ also coincides with $\mathcal{F}_{\alpha}\left(\phi, c^{j}\right)$ in $(8)$ whenever $K(\phi)=0$.

- Next we introduce the dual function, which is the mapping $Q: L_{2}(\Omega) \times \mathbb{R}_{+} \rightarrow \mathbb{R}$ defined by $Q(\lambda, \mu):=\inf _{\left(\phi, c^{j}\right) \in L_{2}(\Omega) \times \mathbb{R}^{2}} \mathcal{G}_{L, \alpha}\left(\phi, c^{j} ; \lambda, \mu\right)$.

Remark 3. Using the definitions above, it is immediate to see that the constrained optimization problem (8) is equivalent to the (primal) problem $\min _{\left(\phi, c^{j}\right)} \widetilde{\mathcal{F}}_{\alpha}\left(\phi, c^{j}\right)$. The corresponding dual problem reads: $\max _{(\lambda, \mu)} Q(\lambda, \mu)$. Moreover, the dual function satisfies the identity $Q(\lambda, \mu)=\inf _{z}\{\theta(z)-\rho(z, \lambda, \mu)\}$.

In this framework, weak duality means that $V_{d}:=\sup _{(\lambda, \mu)} Q(\lambda, \mu) \leqslant$ $\inf _{\left(\phi, c^{j}\right)} \widetilde{\mathcal{F}}_{\alpha}\left(\phi, c^{j}\right)=: V_{p}$, while strong duality corresponds to $V_{d}=V_{p}$, where $V_{p}$ and $V_{d}$ denote the optimal values for the primal and dual problem respectively.

In the following definition, the Fenchel-Moreau conjugated and biconjugated functions are introduced. These functions furnish a natural way to verify weak and strong duality, as we shall see below. The second part of the definition concerns abstract subgradients, which are needed in the proof of our strong duality result.

5 For non-convex constrained optimization problems, a nonzero duality gap may occur when an ordinary Lagrangian functional is used $[43,35]$. 
Definition 2 ([35, chapter 11]). The Fenchel-Moreau conjugated and biconjugated functions of $\theta$ with respect to the coupling function $\rho$ are defined respectively by

$\theta^{\rho}(\lambda, \mu)=\sup _{z \in L_{2}(\Omega)}\{\rho(z, \lambda, \mu)-\theta(z)\} \quad$ and $\quad \theta^{\rho \rho}(z)=\sup _{(\lambda, \mu) \in L_{2}(\Omega) \times \mathbb{R}_{+}}\left\{\rho(z, \lambda, \mu)-\theta^{\rho}(\lambda, \mu)\right\}$.

Moreover, given $\epsilon \geqslant 0$, an element $(\lambda, \mu) \in L_{2}(\Omega) \times \mathbb{R}_{+}$is called $\epsilon$-abstract subgradient of $\theta$ at $\bar{z}$ with respect to $\rho$ when $\theta(z)-\rho(z, \lambda, \mu) \geqslant \theta(\bar{z})-\rho(\bar{z}, \lambda, \mu)-\varepsilon$, for all $z \in L_{2}(\Omega)$. The set of all $\varepsilon$-abstract subgradients of $\theta$ at $\bar{z}$ is called $\epsilon$-subdifferential of $g$ at $\bar{z}$ and is denoted by $\partial_{\rho, \varepsilon} \theta(\bar{z})$.

Remark 4. Note that the perturbation function $\theta$ is lsc at $z=0$. Moreover, from the definitions of $\theta^{\rho}$ and $\theta^{\rho \rho}$ it follows that $\operatorname{dom}\left(\theta^{\rho}\right) \neq \emptyset, \theta^{\rho}(\lambda, \mu)=-Q(\lambda, \mu)$ and $\theta^{\rho \rho}(z) \leqslant \theta(z)$.

In the following lemma, we prove some regularity properties of $\rho$.

Lemma 5. The following assertions hold true.

(i) For any $(\lambda, \mu) \in L_{2}(\Omega) \times \mathbb{R}_{+}$the function $\rho(\cdot, \lambda, \mu)$ is upper semi-continuous at 0 and satisfies $\rho(0, \lambda, \mu)=0$.

(ii) For every neighborhood $V \subset L_{2}(\Omega)$ of $z=0$ and for every $(\lambda, \bar{\mu}) \in L_{2}(\Omega) \times \mathbb{R}_{+}$, it holds (a) $A_{\lambda, \bar{\mu}}^{V}(\mu):=\inf _{z \in V^{c}}\{\rho(z, \lambda, \bar{\mu})-\rho(z, \lambda, \mu)\}>0, \forall \mu>\bar{\mu}$;

(b) $\lim _{\mu \rightarrow \infty} A_{\lambda, \bar{\mu}}^{V}(\mu)=\infty$.

Proof. The continuity of $\rho$ as well as the property $\rho(0, \lambda, \mu)=0$ follow from the definition of the coupling function, proving assertion (i). Assertion (ii) is a consequence of the identity $\rho(z, \lambda, \bar{\mu})-\rho(z, \lambda, \mu)=(\mu-\bar{\mu})\|z\|_{L_{2}}$.

Lemma 6. The weak duality property holds true, i.e. $V_{d} \leqslant V_{p}$.

Proof. From lemma 5 we know that $\rho(0, \lambda, \mu)=0$. Using this fact in definition 2 , we conclude that $\theta^{\rho \rho}(0)=V_{d}$. On the other hand, $\theta(0)=V_{p}$. The proof follows now from remark 4 .

From the above proof, we conclude that weak duality is equivalent to $\theta^{\rho \rho}(0) \leqslant \theta(0)$. Analogously, strong duality (or zero duality gap) can be shown to be equivalent to the identity $\theta^{\rho \rho}(0)=\theta(0)$. This fact is used to prove the first main result of this section, which is stated in theorem 9 after verifying two auxiliary results.

Lemma 7. If $\left(\lambda, \mu_{0}\right) \in \partial_{\rho, \varepsilon} \theta(0)$, then $(\lambda, \mu) \in \partial_{\rho, \varepsilon} \theta(0)$, for every $\mu \geqslant \mu_{0}$.

Proof. The assertion follows from the definition of the $\epsilon$-abstract subgradient $\partial_{\rho, \varepsilon} \theta(\cdot)$, together with lemma 5 (i) and the fact that $\rho(z, \lambda, \cdot)$ is a monotone decreasing function.

Lemma 8. The following assertions hold true.

(a) For all $\varepsilon>0$ it holds $\partial_{\rho, \varepsilon} \theta(0) \neq \emptyset$.

(b) Let $(\bar{\lambda}, \bar{\mu}) \in \operatorname{dom}\left(\theta^{\rho}\right)$ be given. For every $\varepsilon>0$ there exists a $\mu_{0}=\mu_{0}(\varepsilon)$ such that $(\bar{\lambda}, \mu) \in \partial_{\rho, \varepsilon} \theta(0)$, for all $\mu \geqslant \mu_{0}$.

Proof. We already observed that $\theta$ is lsc at $z=0$, as well as $\operatorname{dom}\left(\theta^{\rho}\right) \neq \emptyset$ (see remark 4). These facts together with lemma 5 guarantee the assumptions of [30, theorem 5.2.1], from which the desired results follow. 
Theorem 9. The Lagrangian functional $\mathcal{G}_{L, \alpha}$ has no duality gaps.

Proof. The weak duality property was already established in lemma 6 . Therefore, in order to prove strong duality, it remains to verify that $\theta^{\rho \rho}(0) \geqslant \theta(0)$.

Let $\varepsilon>0$ be given. Lemma 8 guarantees the existence of an element $\left(\bar{\lambda}, \mu_{\varepsilon}\right) \in \partial_{\rho, \varepsilon} \theta(0)$. Thus, from the definition of $\varepsilon$-abstract subgradients, it follows that

$\theta(z)-\rho\left(z, \bar{\lambda}, \mu_{\varepsilon}\right) \geqslant \theta(0)-\rho\left(0, \bar{\lambda}, \mu_{\varepsilon}\right)-\varepsilon=\theta(0)-\varepsilon, \forall z \in L_{2}(\Omega)$.

Hence, it follows from lemma 5 that

$$
\begin{aligned}
\theta^{\rho \rho}(0)=\sup _{(\lambda, \mu)}\left\{\rho(0, \lambda, \mu)-\theta^{\rho}(\lambda, \mu)\right\}=\sup _{(\lambda, \mu)}\left\{-\theta^{\rho}(\lambda, \mu)\right\} \\
\geqslant-\theta^{\rho}\left(\bar{\lambda}, \mu_{\varepsilon}\right)=\inf _{z}\left\{\theta(z)-\rho\left(z, \bar{\lambda}, \mu_{\varepsilon}\right)\right\} \geqslant \theta(0)-\varepsilon .
\end{aligned}
$$

Since $\varepsilon>0$ is arbitrary, the desired inequality follows.

In what follows, we concentrate on the second main result of this section, namely the existence of multipliers. The precise definition of 'generalized Lagrangian multipliers' follows

Definition 3. (Exact penalty representation) A vector $\overline{\bar{\lambda}} \in L_{2}(\Omega)$ is said to support an exact penalty representation for the problem of minimizing $\widetilde{\mathcal{F}}_{\alpha}$ if there exists a $\mu_{0}>0$ such that

$\theta(0)=Q(\bar{\lambda}, \mu)$ and $\operatorname{argmin}_{\left(\phi, c^{j}\right)} \widetilde{\mathcal{F}}_{\alpha}\left(\phi, c^{j}\right)=\operatorname{argmin}_{\left(\phi, c^{j}\right)} \mathcal{G}_{L, \alpha}\left(\phi, c^{j} ; \bar{\lambda}, \mu\right)$,

for all $\mu>\mu_{0}$. (Alternatively, such a vector $\bar{\lambda}$ is said to support an exact penalty representation for the problem of minimizing $\mathcal{F}_{\alpha}$ under the constraint $K(\phi)=0$.)

A proof of existence of generalized multipliers in the sense of definition 3 is given in theorem 11, which is stated after the verification of an important auxiliary result.

Lemma 10. Let $(\bar{\lambda}, \bar{\mu}) \in \operatorname{dom}\left(\theta^{\rho}\right)$ be given. There exists $\hat{\mu}>0$ satisfying

$$
\theta(z) \geqslant \theta(0)-\langle\bar{\lambda}, z\rangle-\hat{\mu}\|z\|_{L_{2}}, \forall z \in L_{2}(\Omega) .
$$

Proof. The existence of an element $(\bar{\lambda}, \bar{\mu}) \in \operatorname{dom}\left(\theta^{\rho}\right)$ follows as in the proof of lemma 8 . Define $\hat{\mu}:=\max \left\{1,\|\bar{\lambda}\|_{L_{2}}\right\}$. Thus, the estimate $-\langle\bar{\lambda}, z\rangle-\hat{\mu}\|z\|_{L_{2}} \leqslant\left(\|\bar{\lambda}\|_{L_{2}}-\hat{\mu}\right)\|z\|_{L_{2}} \leqslant 0$ holds true for all $z \in L_{2}(\Omega)$. Now, we conclude from (15) that $\theta(z) \geqslant \theta(0) \geqslant \theta(0)-\langle\bar{\lambda}, z\rangle-$ $\hat{\mu}\|z\|_{L_{2}}$, for all $z \in L_{2}(\Omega)$.

Theorem 11. There exists a $\bar{\lambda} \in L_{2}(\Omega)$ supporting an exact penalty representation for the problem of minimizing $\mathcal{F}_{\alpha}$ under the constraint $K(\phi)=0$.

Proof. Note that: (i) the set of optimal solutions of the primal problem is nonempty (consequence of assumption (A4)); (ii) the dualizing parametrization function $f\left(\phi, c^{j} ; \cdot\right)$ is lsc at $z=0$ for every $\left(\phi, c^{j}\right)$ (follows from the definition of $f$ ); (iii) the perturbation function $\rho$ is lsc at $z=0$ (see remark 4); (iv) There exists an element $(\bar{\lambda}, \bar{\mu}) \in \operatorname{dom}\left(\theta^{\rho}\right)$ (see remark 4).

These facts together with lemma 5 guarantee the assumptions of [30, theorem 5.2.5]. According to this theorem, the existence of $\bar{\lambda} \in L_{2}(\Omega)$ supporting an exact penalty representation for the problem of minimizing $\widetilde{\mathcal{F}}_{\alpha}$ is equivalent to the existence of an open neighborhood $W \subset L_{2}(\Omega)$ of 0 and some $\mu_{0}>0$ such that $\theta(z) \geqslant \theta(0)+\rho\left(z, \bar{\lambda}, \mu_{0}\right)$, for all $z \in W$. However, due to lemma 10 , this last inequality is satisfied at any neighborhood $W \subset L_{2}(\Omega)$ of 0 . 
Remark 5. The first identity in (16) is equivalent to $\inf _{\left(\phi, c^{j}\right)} \widetilde{\mathcal{F}}_{\alpha}\left(\phi, c^{j}\right)=$ $\inf _{\left(\phi, c^{j}\right)} \mathcal{G}_{L, \alpha}\left(\phi, c^{j} ; \bar{\lambda}, \mu\right)$. Thus, the existence of a vector $\bar{\lambda} \in L_{2}(\Omega)$ satisfying definition 3 is sufficient to guarantee that the problems of minimizing the functionals $\widetilde{\mathcal{F}}_{\alpha}(\cdot, \cdot)$ and $\mathcal{G}_{L, \alpha}(\cdot, \cdot ; \bar{\lambda}, \mu)$ over $\left(\phi, c^{j}\right) \in L_{2}(\Omega) \times \mathbb{R}^{2}$ are equivalent (i.e. both functionals have the same minimizers are the same as well as the same minimal values). Consequently, optimal solutions of the constrained optimization problem (8) can be obtained by solving the unconstrained optimization problemmin ${ }_{\left(\phi, c^{j}\right)} \mathcal{G}_{L, \alpha}\left(\phi, c^{j} ; \bar{\lambda}, \mu\right)$. However, a pair $\left(\bar{\lambda}, \mu_{0}\right)$ satisfying definition 3 has to be found first.

Another consequence of the first identity in (16) is the following fact:

$$
V_{p}=\theta(0)=Q(\bar{\lambda}, \mu) \leqslant \sup _{(\lambda, \mu)} Q(\lambda, \mu)=V_{d} .
$$

From this inequality and the weak duality property established in lemma 6 , the strong duality property follows. Therefore, theorem 9 can be interpreted as a corollary of theorem 11 .

Note that the existence of (generalized) multipliers justifies the implementation of dual algorithms in order to approximate the solutions of the constrained optimization problem in (8). These algorithms allow the simultaneous determination of the optimal solution as well as the generalized multipliers.

\subsection{An iterative algorithm based on the augmented Lagrangian method}

In the following, we propose a numerical method based on the PCLS approach and augmented Lagrangian for solving the inverse problem in (5). This is an iterative method which exploits the definition of abstract multipliers in definition 3, generating a sequence of approximate solutions to the constrained optimization problem in (8).

More precisely, our method can be interpreted as an Usawa-type iteration [21, 25, 35, 26], which aims to find an $L_{2}$-vector $\bar{\lambda}$ satisfying definition 3 and (simultaneously) find a pair $\left(\bar{\phi}, \bar{c}^{j}\right) \in \operatorname{argmin}_{\left(\phi, c^{j}\right)} \mathcal{G}_{L, \alpha}\left(\phi, c^{j} ; \bar{\lambda}, \mu\right)$.

Note that in definition 3 any $\mu$ large enough (namely $\mu>\mu_{0}$ ) can be used to characterize $\bar{\lambda} \in L_{2}(\Omega)$ as a vector supporting an exact penalty representation for the problem of minimizing $\mathcal{F}_{\alpha}$ under the constraint $K(\phi)=0$. Therefore, in our algorithm we do not update the value of the $\mu$ along the iteration. The outcome of our numerical experiments are not strongly influenced by the choice the (constant) penalty factor $\mu$.

Starting with initial guesses $\left(\phi_{0}, c_{0}^{j} ; \lambda_{0}\right)$ and $\mu>0$ sufficiently large, the proposed iterative method consists essentially of two parts: (i) given the current iterate $\left(\phi_{k}, c_{k}^{j} ; \lambda_{k}\right)$, the update of the components $\left(\phi_{k}, c_{k}^{j}\right)$ is computed through the minimization of $\mathcal{G}_{L, \alpha}\left(\cdot, \cdot, \lambda_{k}, \mu\right)$ with respect to $\left(\phi, c^{j}\right)$, i.e.

$$
\left(\phi_{k+1}, c_{k+1}^{j}\right):=\arg \min _{\left(\phi, c^{j}\right)} \mathcal{G}_{L, \alpha}\left(\phi, c^{j} ; \lambda_{k}, \mu\right),
$$

(ii) the Lagrange multiplier $\lambda_{k}$ is updated giving a gradient step of $\mathcal{G}_{L, \alpha}\left(\phi_{k+1}, c_{k+1}^{j}, \cdot, \mu\right)$ with respect to $\lambda$, i.e.

$$
\lambda_{k+1}:=\lambda_{k}+\mu K\left(\phi_{k+1}\right) .
$$

In our numerical implementations, we follow the approach in [32]. A solution of the minimization problem (i) is approximated by solving the set of optimality conditions

$$
\frac{\partial}{\partial \phi} \mathcal{G}_{L, \alpha}\left(\phi, c^{j} ; \lambda_{k}, \mu\right)=0, \quad \frac{\partial}{\partial c^{j}} \mathcal{G}_{L, \alpha}\left(\phi, c^{j} ; \lambda_{k}, \mu\right)=0 .
$$

For the implementation of step (ii), this is a simple gradient step with respect to $\lambda$ of the functional $\mathcal{G}_{L, \alpha}$ which can be computed in an explicit way. 
Remark 6. In order to solve the set of optimality conditions in (19), an artificial time variable $t$ is introduced and the PDE

$$
\frac{\partial \phi}{\partial t}=\frac{\partial}{\partial \phi} \mathcal{G}_{L, \alpha}\left(\phi, c^{j} ; \lambda_{k}, \mu\right), \quad \frac{\partial c^{j}}{\partial t}=\frac{\partial}{\partial c^{j}} \mathcal{G}_{L, \alpha}\left(\phi, c^{j} ; \lambda_{k}, \mu\right)=0,
$$

is solved until it reaches a steady solution. This steady solution is also a solution of the optimality conditions (19) $[32,33]$.

In the numerical realizations presented in section 5, we use a forward Euler method for calculating a steady solution of the PDE above. This computational scheme corresponds to an inner iteration for updating the pair $\left(\phi, c^{j}\right)$ :

\section{Numerical experiments: setup and algorithms}

In this section, we discuss the numerical implementations of iterative methods based on the approaches discussed in sections 2 and 3. As a benchmark problem we consider the inverse potential problem (IPP), which is similar to the one considered in [20, 39, 13, 22, 40].

It is worth noting that generalizations of this inverse problem have applications in inverse gravimetry, EEG, ECG and EMG (see [22-24, 31, 38-41] and the references therein).

\subsection{The inverse potential problem}

The forward problem consists of solving on a given Lipschitz domain $\Omega \subset \mathbb{R}^{n}$, for a given source function $u \in L_{2}(\Omega)$, the Poisson boundary value problem

$$
-\Delta w=u, \text { in } \Omega, \quad w=0 \text { on } \partial \Omega .
$$

This problem can be modeled by the operator $F: L_{2}(\Omega) \rightarrow L_{2}(\partial \Omega), F(u):=\left.w_{\nu}\right|_{\partial \Omega}[24]$, where $w \in H_{0}^{1}(\Omega)$ is the unique solution of (20). The corresponding inverse problem is the socalled IPP, which consists of recovering an $L_{2}$-function $u$, from measurements of the Cauchy data of its corresponding potential on the boundary of $\Omega$.

Using this notation, the IPP can be written in the abbreviated form $F(u)=y^{\delta}$, where the available noisy data $y^{\delta} \in L_{2}(\partial \Omega)$ have the same meaning as in (2).

It is worth noting that this inverse problem has, in general, non-unique solution [22]. Sufficient conditions for identifiability are given in [23]. For issues related to redundancy of data as well as for an example of non-identifiability we refer the reader to [22]. A generalization of this inverse problem, with the Laplacian replaced by a general elliptic operator, appears in many relevant applications including: inverse gravimetry [31, 24], EEG [15], and EMG [41].

In our experiments, we follow [13] in the experimental setup, selecting $\Omega=(0,1) \times(0,1)$ and assuming that the unknown parameter is a piecewise constant function of the form $u=1+\chi_{D}$, where $D \subset \subset \Omega$. In particular, we allow piecewise constant functions $u$ supported at domains consisting of several connected components. It is worth mentioning that, for this class of parameters, no unique identifiability result is known in the literature [24].

\subsection{An algorithm based on PCLS and Tikhonov method for the IPP}

In the following, we present a numerical algorithm for solving the IPP based on the PCLS approach and the Tikhonov method, cf discussed in section 2.1.

First, a constant $N \in \mathbb{N}$ is chosen in order to control the operator splitting scheme. Each step of this iterative method consists of four parts (see table 2). The first half of the operator splitting scheme consists of parts (1), (2) and (3). The second half of the operator splitting scheme consists of part (4), as described below. 
Table 1. Forward Euler method for updating the pair $\left(\phi, c^{j}\right)$. This inner iteration corresponds to step part (i) of the iterative algorithm based on the augmented Lagrangian method.

1.Take $\left(\tilde{\phi}_{0}, \tilde{c}_{0}\right)=\left(\phi_{k}, c_{k}\right)$; and $\varepsilon>0$ a specified precision.

2.Compute the inner iteration $\tilde{\phi}_{n+1}=\tilde{\phi}_{n}-\Delta_{\phi} \frac{\partial}{\partial \phi} \mathcal{G}_{L, \alpha}\left(\tilde{\phi}_{n}, \tilde{c}_{n}^{j} ; \lambda_{k}, \mu\right), \tilde{c}_{n+1}^{j}=$

$\tilde{c}_{n}^{j}-\Delta_{c^{j}} \frac{\partial}{\partial c^{j}} \mathcal{G}_{L, \alpha}\left(\tilde{\phi}_{n}, c_{n}^{j} ; \lambda_{k}, \mu\right)$, for $n=0, \ldots, n^{\star}$, where $n^{\star} \in \mathbb{N}$ is such that the pair $\left(\tilde{\phi}_{n^{\star}}, \tilde{c}_{n^{\star}}^{j}\right)$ satisfies the stop criteria $\left|\frac{\partial}{\partial \phi} \mathcal{G}_{L, \alpha}\left(\tilde{\phi}_{n^{\star}}, \tilde{c}_{n^{\star}}^{j} ; \lambda_{k}, \mu\right)\right|<\varepsilon$, and $\left|\frac{\partial}{\partial c^{j}} \mathcal{G}_{L, \alpha}\left(\tilde{\phi}_{n^{\star}}, c_{n^{\star}}^{j} ; \lambda_{k}, \mu\right)\right|<\varepsilon$.

3.Take $\left(\phi_{k+1}, c_{k+1}^{j}\right):=\left(\tilde{\phi}_{n^{\star}}, \tilde{c}_{n^{\star}}^{j}\right)$.

Table 2. Iterative algorithm based on the PCLS approach and the Tikhonov method for the IPP.

1. Evaluate the residual $r_{k}:=F\left(P_{p c}\left(\phi_{k}, c_{k}^{j}\right)\right)-y^{\delta}=\left.\left(w_{k}\right)_{v}\right|_{\partial \Omega}-y^{\delta}$, where $w_{k}$ solves

$$
\Delta w_{k}=P_{p c}\left(\phi_{k}, c_{k}^{j}\right) \text {, in } \Omega ; \quad w_{k}=0 \text {, at } \partial \Omega \text {. }
$$

2. Evaluate $h_{k}:=F^{\prime}\left(P_{p c}\left(\phi_{k}, c_{k}^{j}\right)\right)^{*}\left(r_{k}\right) \in L_{2}(\Omega)$, solving

$$
\Delta h_{k}=0 \text {, in } \Omega ; \quad h_{k}=r_{k} \text {, at } \partial \Omega \text {. }
$$

3. Update $\phi_{k}, c_{k}^{j}$ according to (11), (12) and calculate $\phi_{k+1 / 2}, c_{k+1 / 2}^{j}$ from

$$
\begin{aligned}
& \phi_{k+1 / 2}:=\phi_{k}-\left(c_{k}^{2}-c_{k}^{1}\right) h_{k}-\frac{\alpha}{2}\left(c_{k}^{2}-c_{k}^{1}\right) s_{k}, \\
& c_{k+1 / 2}^{1}:=c_{k}^{j}-\int_{\Omega} \phi_{k} h_{k}-\frac{\alpha}{2} \int_{\Omega}\left(1-\phi_{k}\right) s_{k},
\end{aligned}
$$$$
c_{k+1 / 2}^{2}:=c_{k}^{j}-\int_{\Omega} \phi_{k} h_{k}-\frac{\alpha}{2} \int_{\Omega} \phi_{k} s_{k},
$$

where $s_{k}:=\nabla \cdot\left[\nabla P_{p c}\left(\phi_{k}, c_{k}^{j}\right) /\left|\nabla P_{p c}\left(\phi_{k}, c_{k}^{j}\right)\right|\right]$.

4. If $(k \bmod N)=0$ then

Update $\phi_{k+1 / 2}, c_{k+1 / 2}^{j}$ according to (13), (14) and calculate $\phi_{k+1}, c_{k+1}^{j}$ Else

Take $\phi_{k+1}=\phi_{k+1 / 2}, c_{k+1}^{j}=c_{k+1 / 2}^{j}$.

(1) The residual $r_{k} \in L_{2}(\partial \Omega)$ of the iterate $\left(\phi_{k}, c_{k}^{j}\right)$ is evaluated (this requires solving one elliptic BVP of Dirichlet type).

(2) The $L_{2}$-solution $h_{k}$ of the adjoint problem for the residual is evaluated (this corresponds to solving one elliptic BVP of Dirichlet type).

(3) The level-set function $\phi_{k}$ and the level values $c_{k}^{j}$ are updated according to the first part of the operator splitting scheme (i.e. with respect to the Functional $\mathcal{G}_{\alpha}^{1}$ ); this results in the computation of $\phi_{k+1 / 2}, c_{k+1 / 2}^{j}$.

(4) If the iteration counter $k$ is not a multiple of $N$, then skip the second part of the operator splitting scheme and set $\phi_{k+1}:=\phi_{k+1 / 2}, c_{k+1}^{j}:=c_{k+1 / 2}^{j}$.

Otherwise, the level-set function $\phi_{k+1 / 2}$ and the level values $c_{k+1 / 2}^{j}$ are updated according to the second part of the operator splitting scheme (i.e. with respect to the functional $\mathcal{G}_{\alpha}^{2}$ ); this allows the computation of $\phi_{k+1}, c_{k+1}^{j}$.

\subsection{An algorithm based on PCLS and augmented Lagrangian for the IPP}

In what follows, we present a numerical algorithm for solving the IPP based on the PCLS approach and the augmented Lagrangian method, cf discussed in subsection 3.1.

First, an initial guess $\left(\phi_{0}, c_{0}^{j} ; \lambda_{0}\right)$ is chosen for the level-set function, the level values, and Lagrange multiplier. Each step of this iterative method consists of two parts (see table 3).

(1) The first part, consists of the inner iteration (1.1)-(1.5) and corresponds to the forward Euler method described in table 1 for approximating a minimizer of the functional $\mathcal{G}_{L, \alpha}\left(\cdot, \cdot, \lambda_{k}\right)$ in (17). This inner iteration starts at $\left(\tilde{\phi}_{0}, \tilde{c}_{0}^{j}\right):=\left(\phi_{k}, c_{k}^{j}\right)$ and produces a sequence $\left\{\left(\tilde{\phi}_{n}, \tilde{c}_{n}^{j}\right)\right\}$. The calculation of this sequence is stopped at step $n=n^{\star}$, when $\left(\tilde{\phi}_{n^{\star}}, \tilde{c}_{n^{\star}}^{j}\right)$ solves the optimality conditions (19) up to an a priori chosen precision $\varepsilon>0$. 
Table 3. Iterative algorithm based on the PCLS approach and augmented Lagrangian method for the IPP.

1. Update of the pair $\left(\phi_{k}, c_{k}^{j}\right)$ :

1.1 Take $n=0$ and $\left(\tilde{\phi}_{0}, \tilde{c}_{0}^{j}\right):=\left(\phi_{k}, c_{k}^{j}\right)$;

1.2 Evaluate the residual $r_{n}:=F\left(P_{p c}\left(\tilde{\phi}_{n}, \tilde{c}_{n}^{j}\right)\right)-y^{\delta}=\left.\left(w_{n}\right)_{v}\right|_{\partial \Omega}-y^{\delta}$, where $w_{n}$ solves

$\Delta w_{n}=P_{p c}\left(\tilde{\phi}_{n}, \tilde{c}_{n}^{j}\right)$, in $\Omega ; \quad w_{n}=0$, at $\partial \Omega$.

1.3 Evaluate $h_{n}:=F^{\prime}\left(P_{p c}\left(\tilde{\phi}_{n}, \tilde{c}_{n}^{j}\right)\right)^{*}\left(r_{n}\right) \in L_{2}(\Omega)$, solving

$\Delta h_{n}=0$, in $\Omega ; \quad h_{n}=r_{n}$, at $\partial \Omega$.

$1.4 U$ pdate $\tilde{\phi}_{n}, \tilde{c}_{n}^{j}$ from

$\tilde{\phi}_{n+1}:=\tilde{\phi}_{n}-\left(\tilde{c}_{n}^{2}-\tilde{c}_{n}^{1}\right) h_{n}-\frac{\alpha}{2}\left(\tilde{c}_{n}^{2}-\tilde{c}_{n}^{1}\right) s_{n}-\lambda_{k} K\left(\tilde{\phi}_{n}\right)$,

$\tilde{c}_{n+1}^{1}:=\tilde{c}_{n}^{j}-\int_{\Omega}\left(1-\tilde{\phi}_{n}\right) h_{n}-\frac{\alpha}{2} \int_{\Omega}\left(1-\tilde{\phi}_{n}\right) s_{n}$,

$\tilde{c}_{n+1}^{2}:=\tilde{c}_{n}^{j}-\int_{\Omega} \tilde{\phi}_{n} h_{n}-\frac{\alpha}{2} \int_{\Omega} \tilde{\phi}_{n} s_{n}$

where $s_{n}:=\nabla \cdot\left[\nabla P_{p c}\left(\tilde{\phi}_{n}, \tilde{c}_{n}^{j}\right) /\left|\nabla P_{p c}\left(\tilde{\phi}_{n}, \tilde{c}_{n}^{j}\right)\right|\right]$.

1.5 If $\left|\frac{\partial}{\partial \phi} \mathcal{G}_{L, \alpha}\left(\tilde{\phi}_{n}, \tilde{c}_{n}^{j} ; \lambda_{k}\right)\right|<\varepsilon$ and $\left|\frac{\partial}{\partial c^{j}} \mathcal{G}_{L, \alpha}\left(\tilde{\phi}_{n}, \tilde{c}_{n}^{j} ; \lambda_{k}\right)\right|<\varepsilon$

Then $n^{\star}:=n$; update $\left(\phi_{k+1}, c_{k+1}^{j}\right)=\left(\tilde{\phi}_{n^{\star}}, \tilde{c}_{n^{\star}}^{j}\right)$

Else $n:=n+1$; go to $\mathbf{1 . 2} 2$. Update the Lagrange multiplier $\lambda_{k+1}=\lambda_{k}+\mu K\left(\phi_{k+1}\right)$.

Note that $\lambda_{k}$ remains fixed during this inner iteration. When $n=n^{\star}$ is reached we update $\left(\phi_{k+1}, c_{k+1}^{j}\right)=\left(\tilde{\phi}_{n^{\star}}, \tilde{c}_{n^{\star}}^{j}\right)$.

(2) The second part of the iterative step corresponds to the explicit gradient step in (18).

Note that, for each $\lambda_{k}$, this inner iteration corresponds to a full run of the algorithm in table 2. At a first glance, this may be seen as a draw back of this algorithm based on the augmented Lagrangian method. However, some relevant facts should be observed at this point.

- Our numerical experiments show that the overall performance of the method is not influenced by the fact that the functional $\mathcal{G}_{L, \alpha}\left(\cdot, \cdot, \lambda_{k}\right)$ in (17) is not precisely minimized in each realization of step part (1). Therefore, accurate results can be computed using relatively large values of $\varepsilon$ in 1.5 (see table 3 ).

- Moreover, differently from the algorithm in table 2, there is no need to use the operator splitting scheme in the implementation of the inner iteration. Therefore, it takes the inner iteration in step part (1) only a small number of steps to enforce the constraint $\mathcal{K}\left(\tilde{\phi}_{n}\right)=0$.

Consequently, the number $n^{\star}$ of steps effectively computed in (1.1)-1.5) remains small along the outer iteration ${ }^{6}$, and computational effort needed to perform each step of the algorithm in table 3 is comparable to the effort needed to execute one step of the algorithm in table 2.

\section{Numerical experiments: IPP with exact and noisy data}

\subsection{First numerical example: exact data}

In this first numerical experiment, we aim to identify the right-hand side $u$ of (20) from the knowledge of the exact data $y=\left.w_{\nu}\right|_{\partial \Omega}$. We assume that the level values $c^{1}=0, c^{2}=1$ are given and only the support of $u$ has to be identified.

The numerical investigation of this slightly simpler setup of the IPP (with known level values $c^{j}$ ) proved to be rich enough to establish a comparison between the performance of the algorithms discussed in sections 2 and 3, as we shall see below.

The exact data $y=F(u)$ is obtained by solving numerically the elliptic boundary value problem in (20) at a very fine grid (the word 'exact' here means: up to the precision of the

6 In the computations presented in section $5, n^{\star}$ was never larger than 5 . 


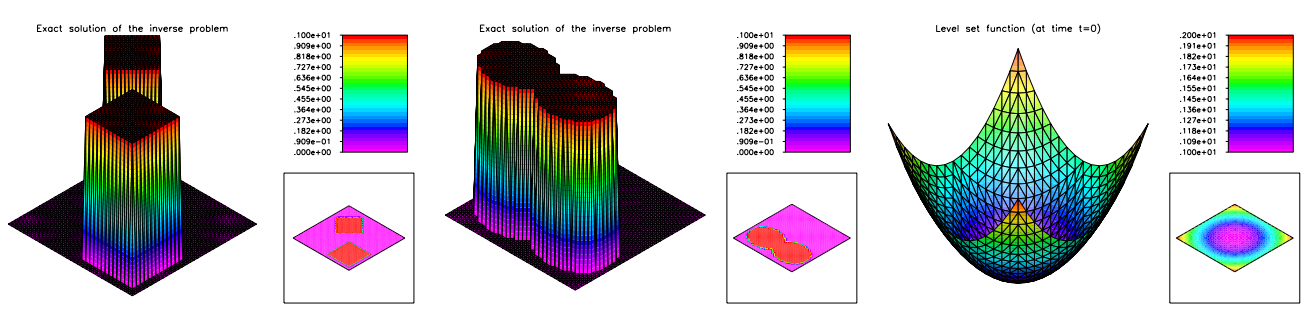

Figure 1. First experiment: the picture on the left-hand side shows the coefficient $u_{\text {exact }}$ to be reconstructed in subsections 5.1 and 5.2. The center picture shows the coefficient $u_{\text {exact }}$ to be reconstructed in subsection 5.3. On the right-hand side, the initial configuration for the level-set function used in the implementation of all numerical discussed in this paper.

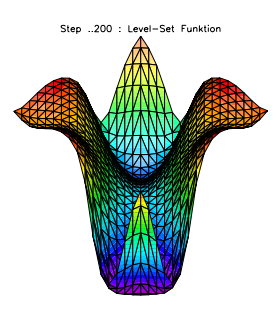

$$
\text { Step. .200 }
$$

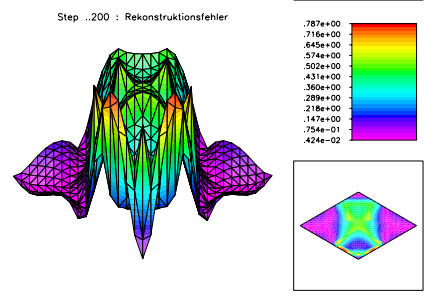

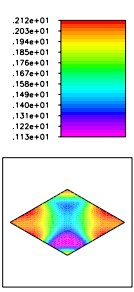
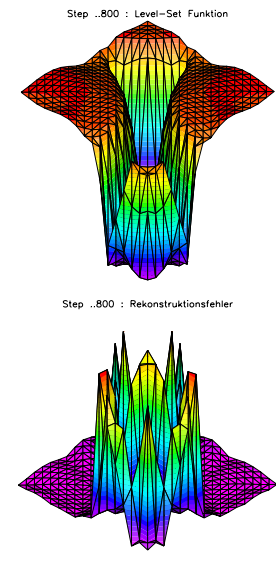
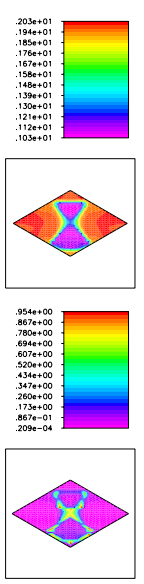
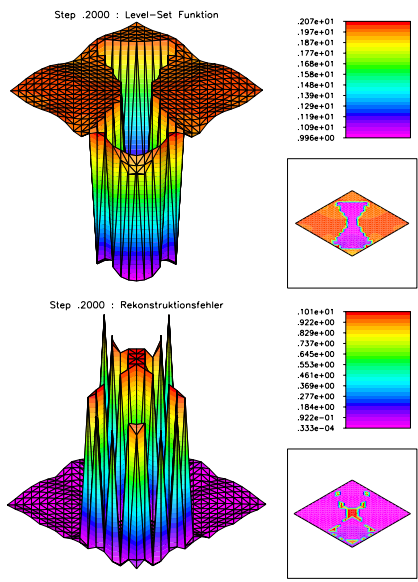

Figure 2. First experiment: PCLS with Tikhonov method and constant $\mu=0.001$. On the first line, plots of $\phi_{k}$, for $k=200,800,2000$, for the operator splitting method. The pictures on the second line show the corresponding iteration error.

numerical method used for solving the direct problem). In order to avoid inverse crimes, the direct problem (20) is solved on an adaptively refined finite element grid with 8.804 nodes. On the other hand, in the numerical implementation of our iterative methods, all boundary value problems are solved at an uniform grid with 545 nodes (33 nodes at each boundary side).

In figure 1 , the solution $u_{\text {exact }}$ of the inverse problem is plotted. Moreover, the initial guess for the level-set function $\phi_{0}(x)$ is also plotted (all numerical methods implemented below use this function $\phi_{0}$ as initial guess). Note that the support of $u_{\text {exact }}$ is a non-connected proper subset of $\Omega$, what represents a challenge for most classical level-set methods.

In order to test the performance of the Tikhonov method with operator splitting scheme (see subsection 2.1), two distinct strategies of choice for the parameter $\mu$ are implemented:

(i) A constant small value $(\mu=0.001)$ is used (see figure 2 ).

(ii) The iterative method is started with $\mu=\mu_{0}=0.001$; and the value of $\mu$ is increased by

$1 \%$ every time the 2 nd part of the operator splitting scheme is called (see figure 3 ).

In both implementations, the constant $N=8$ is chosen to control the frequency of the operator splitting scheme, i.e. the second step of the splitting scheme is called once, after every eight steps of the algorithm using only the first part of the splitting scheme. 


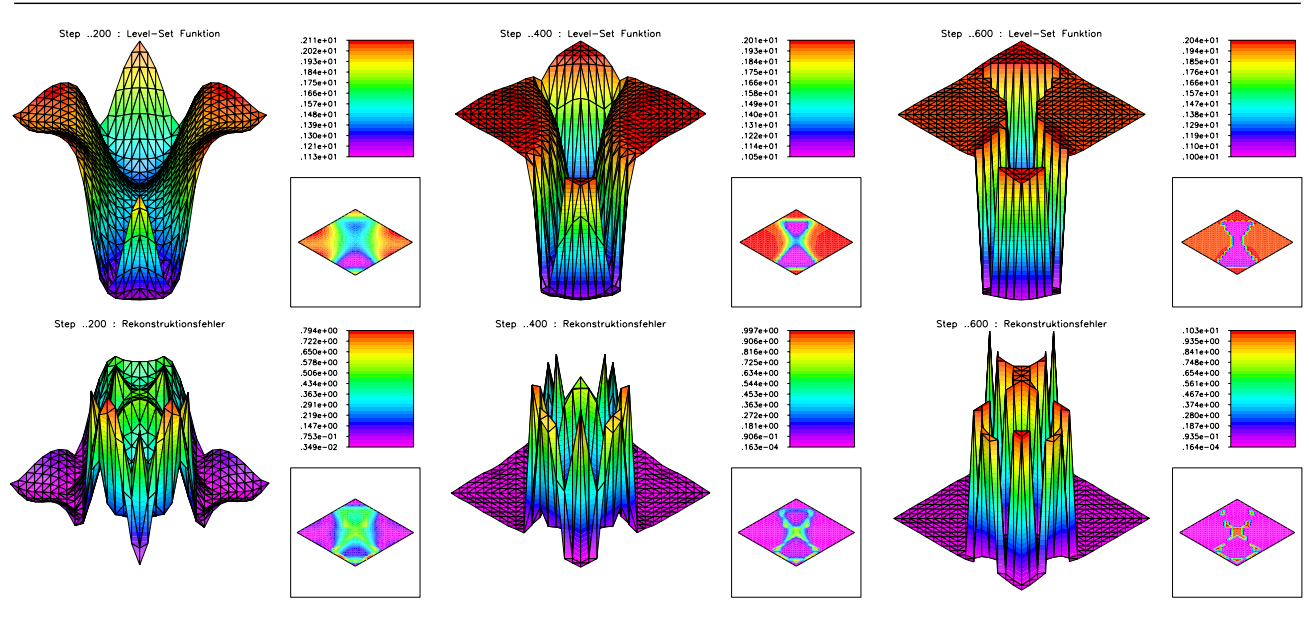

Figure 3. First experiment: PCLS with the Tikhonov method and non-constant $\mu$. On the first line, plots of $\phi_{k}$, for $k=200,400,600$, for the operator splitting method. The pictures on the second line show the corresponding iteration error.

In figure 2, the evolution of the Tikhonov method using the first operator splitting scheme, with constant $\mu$, is presented. The algorithm is stopped when the residual in (7) drops below a specified threshold, namely

$$
\left\|F\left(P_{p c}\left(\phi_{k}, c^{j}\right)\right)-y\right\|_{L_{2}} \leqslant 10^{-2} \text { and }\left\|\mathcal{K}\left(\phi_{k}\right)\right\|_{L_{1}} \leqslant 10^{-3} .
$$

According to our numerical experiments, after reaching this stop criteria (with $k=2000$ ) the iteration stagnates. At this point, two facts should be observed:

- The level-set function $\phi_{2000}$ as well as the corresponding approximate solution $P_{p c}\left(\phi_{2000}, c^{j}\right)$ are 'almost' piecewise constant (see pictures on the right-hand side of figure 2).

- The shapes of the inclusions are not well reconstructed. As a matter of fact, the approximate solutions $P_{p c}\left(\phi_{k}, c^{j}\right)$ produced by this method do not distinguish the existence of two inclusions in the support of the solution $u_{\text {exact }}$.

In figure 3 , the second operator splitting, with non-constant $\mu$, is implemented. Here, the value of $\mu$ is slowly increased along the iteration (see section 2.1). Using this strategy, after only $k=600$ steps this iteration already generates a level-set function $\phi_{k}$ satisfying the stop criteria (21).

Remark 7. The constraint $\left\|\mathcal{K}\left(\phi_{k}\right)\right\|_{L_{1}}=0$ is enforced after a smaller number of iterations if we use the implementation with non-constant $\mu$. However, none of the implementations (with constant/non-constant $\mu$ ) can distinguish between the existence of two inclusions. As a matter of fact, after the stop criteria (21) has been reached, the iteration error $\left|P_{p c}\left(\phi_{k}, c^{j}\right)-u_{\text {exact }}\right|$ is essentially the same for both strategies of choice for the parameter $\mu$.

Remark 8. What concerns is the choice of the constant value $\mu$; some facts should be observed.

(i) If $\mu>0$ is large (close to one), then the condition $\left\|\mathcal{K}\left(\phi_{k}\right)\right\|_{L_{1}} \leqslant 10^{-3}$ is satisfied after a small number of iterations. However, the iteration 'stagnates' after this point and first part of the stop criteria in (21) is never reached.

(ii) If $\mu>0$ is too small (close to zero), then the quality of the final approximate solution obtained with the splitting method is essentially the same as in (i). However, the number of iterations needed to reach (the second part of the) stop criteria (21) increases dramatically. 


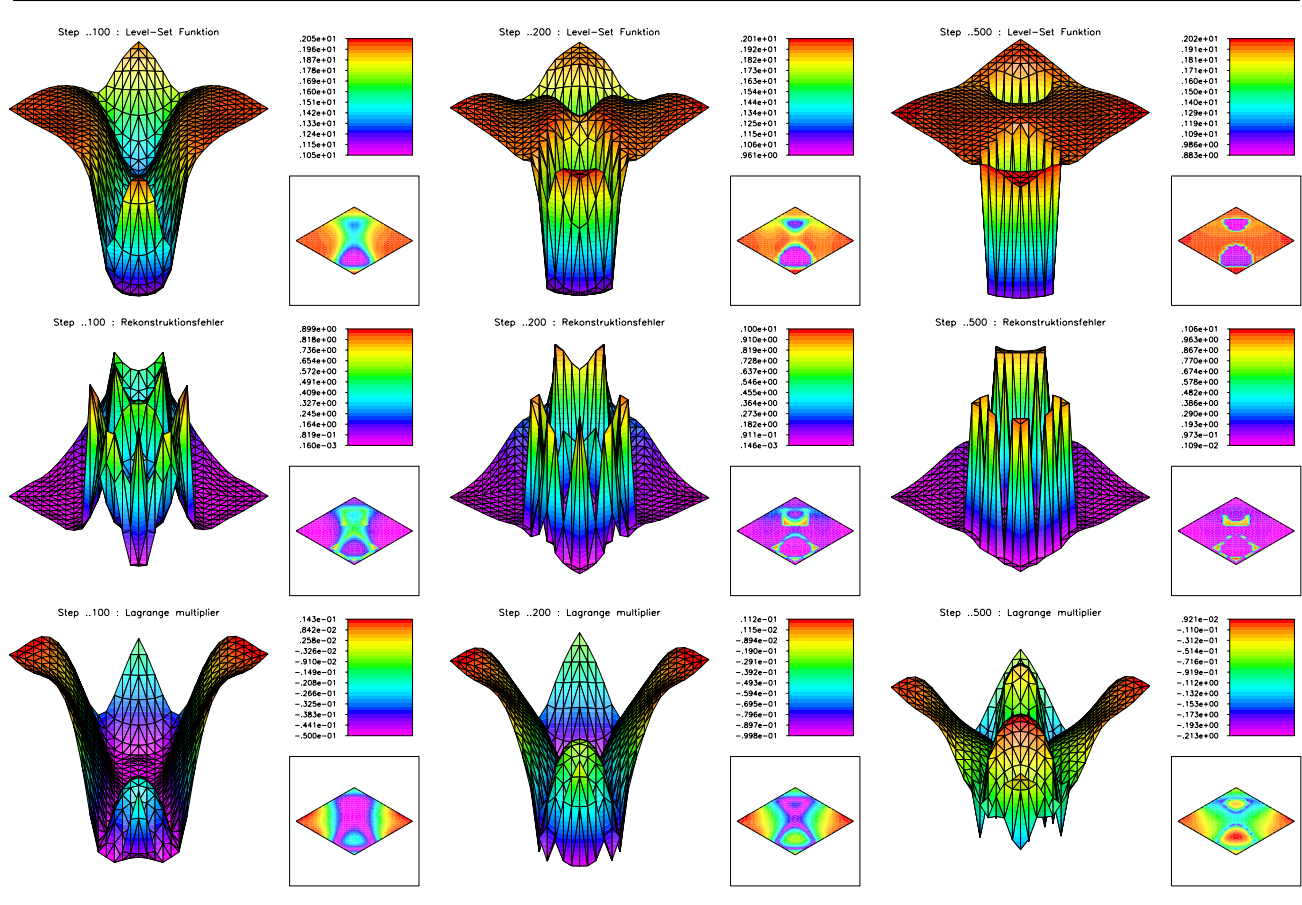

Figure 4. First experiment: PCLS with augmented Lagrange. On the first line, plots of $\phi_{k}$, for $k=100,200,500$. The pictures on the second line show the corresponding iteration error. The pictures on the third line show the corresponding Lagrangian multipliers.

(iii) The constant $N \in \mathbb{N}$ determines how frequently the operator splitting scheme should be activated. In our experiments, the best results were produced with $N \approx 10$. If $N$ is too small (e.g., $N=1$ or $N=2$ ), then the effects in (i) are again observed, no matter the choice of $\mu$. On the other hand, if $N$ is too large (for this particular problem this means $N \gg 10$ ), then the effects in (ii) are observed.

Summing-up. These two experiments indicate that the choice of increasing values of $\mu$ along the iteration indeed produces a faster numerical convergence. Moreover, the approximate solution computed using increasing values of $\mu$ has the same quality as the approximate solution computed using constant values of $\mu$.

This fact motivated us to investigate the method in section 3 as an alternative to obtain more accurate numerical approximations for exponentially ill-posed inverse problems as the IPP.

In what follows, we present a third numerical method for the IPP with exact data: namely the algorithm based on the augmented Lagrangian method for the PCLS approach (see subsection 4.3). In figure 4, the obtained sequence of level-set functions $\phi_{k}$ is plotted, as well as the corresponding iteration errors $\left|P_{p c}\left(\phi_{k}, c^{j}\right)-u_{\text {exact }}\right|$ and the sequence of Lagrangian multipliers $\lambda_{k}$. The initial condition for the level-set function is the one in figure 1, and the initial condition for the Lagrange multiplier reads $\lambda_{0}(x)=0, x \in \Omega$.

The iteration is stopped according to the criteria in (21) (note that the second inequality has to be replaced by $\left\|K\left(\phi_{k}\right)\right\|_{L_{2}} \leqslant 10^{-3}$ ). Nevertheless, the quality of the approximate solution is clearly better (compare figure 4 with figures 2 and 3 ). Not only it is possible to distinguish the 

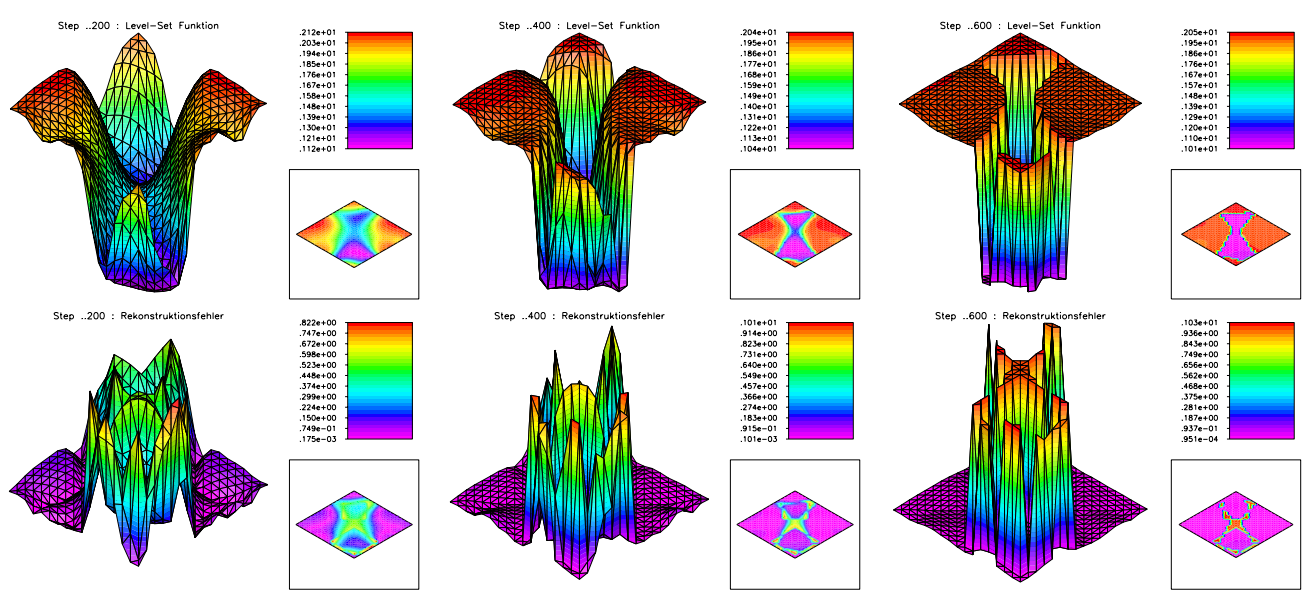

Figure 5. Second experiment: PCLS with Tikhonov method and non-constant $\mu$. Data contaminated with $10 \%$ random noise. On the first line, plots of $\phi_{k}$, for $k=200,400,600$, for the operator splitting method. The pictures on the second line show the corresponding iteration error $\left|P_{p c}\left(\phi_{k}, c^{j}\right)-u_{\text {exact }}\right|$.

two inclusions in support of $u_{\text {exact }}$, but the number of iterations needed to reach the stop criteria (21) is comparable with the fastest operator splitting schemes (the one with non-constant $\mu$ ).

We performed numerous numerical simulations with distinct choices for the initial guess $\phi_{0}$. We observed that the number of iterative steps required in order to obtain an acceptable approximation (up to the same predefined precision) does not depend strongly on the choice of the initial guess $\phi_{0}$.

\subsection{Second numerical example: noisy data}

In the following, we consider the same inverse problem as in subsection 5.1, with the solution shown in figure 1 . This time however, the data $y^{\delta} \in L_{2}(\partial \Omega)$, with $\delta>0$, for the inverse problem are obtained by adding to the exact data $y=F(u)$ randomly generated noise of $10 \%$.

As in the previous experiment, the direct problem is solved at a grid that is finer than the one used in the numerical implementation of the level-set method. The initial guess $\phi_{0}$ is the same as in the previous experiment with exact data (see subsection 5.1). As stop criteria, we used the generalized discrepancy with $\gamma=2$, i.e. the iteration was stopped when for the first time:

$$
\left\|F\left(P_{p c}\left(\phi_{k}, c^{j}\right)\right)-y^{\delta}\right\|_{L_{2}} \leqslant \gamma \delta
$$

In figure 5, we show the results obtained using the PCLS approach and Tikhonov method for non-constant $\mu$. The strategy to increase the values of $\mu$ is the same one used in the previous experiment with exact data (see subsection 5.1). In this figure, we show pictures of the level-set function as well as the corresponding iteration error along the iteration.

In figure 6, we show the results obtained using the PCLS approach with augmented Lagrange for the same set of contaminated data and same initial guess $\phi_{0}$. Pictures of the level-set function and the corresponding iteration error are shown in the first and second lines of this figure. 


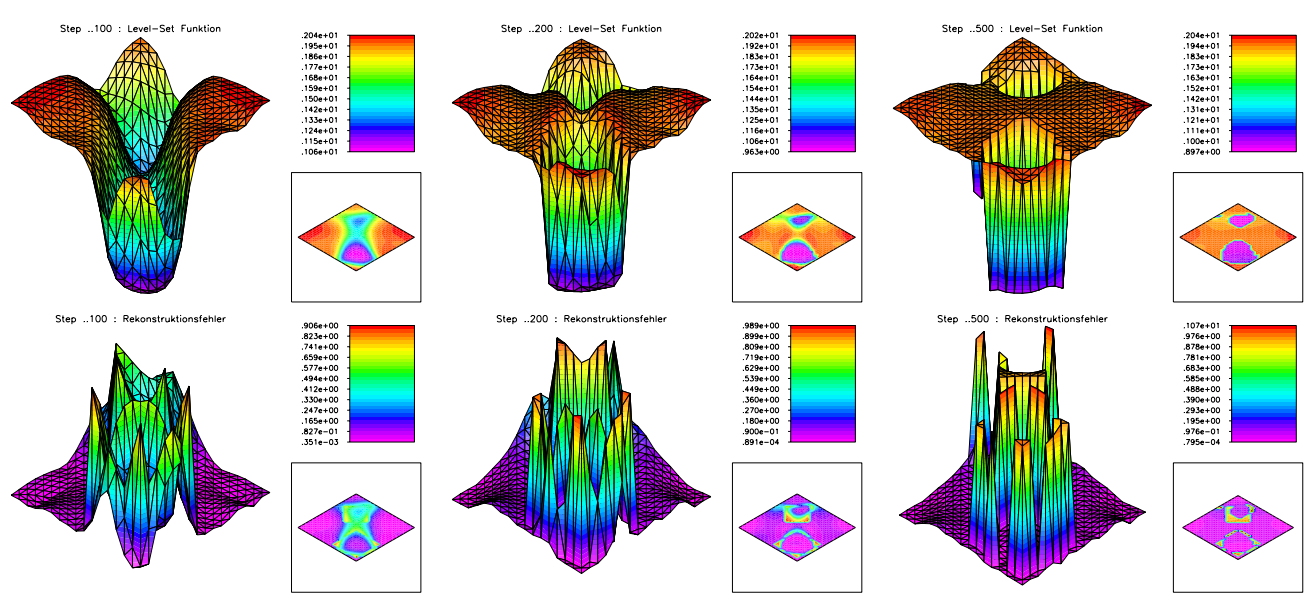

Figure 6. Second experiment: PCLS with augmented Lagrange. Data contaminated with 10\% random noise. On the first line, plots of $\phi_{k}$, for $k=100,200,500$. The pictures on the second line show the corresponding iteration error.

\subsection{Third numerical example: non-convex parameter}

In this last numerical experiment, we consider once again the IPP in (20). Differently from the previous two examples, the solution (unknown parameter) is the non-convex inclusion shown in the center picture of figure 1 . The data $y^{\delta} \in L_{2}(\partial \Omega)$ is assumed to be exact (i.e. $\left.\delta=0\right)$ and the level values $c^{1}=0$ and $c^{2}=1$ are given.

The identification of a similar inclusion using level-set methods was considered by Scherzer et al in [20], who described the slow behavior of the method for identifying the shape of the boundary at points close to the non-convex part of the inclusion.

As in the previous examples, the direct problem (20) is again solved on an adaptively refined finite element grid with 8.804 nodes. However, in the numerical implementation of the iterative methods, all boundary value problems are solved at an uniform grid with 2.280 nodes (64 nodes at each boundary side). This is done in order to better capture the non-convex part of the inclusion.

In figure 7, we show the evolution obtained using the PCLS approach and the Tikhonov method for non-constant $\mu$. The strategy to increase the values of $\mu$ is the same one used in the previous experiments. In this figure, plots of the level-set function as well as plots of the corresponding iteration error along the iteration are shown. In figure 8 , the evolution of the PCLS approach with augmented Lagrange is shown. The number of iterative steps required to obtain an acceptable approximation is similar for both approaches. However, the PCLS method based on the augmented Lagrange approach produced a more accurate approximate solution, specially what concerns the shape of the inclusion at the critical part of the boundary.

\section{Conclusions}

In this paper, a PCLS framework is proposed for representing the parameter space of ill-posed problems with piecewise constant solutions. Two distinct approaches for solving the resulting operator equation (5) are proposed. 

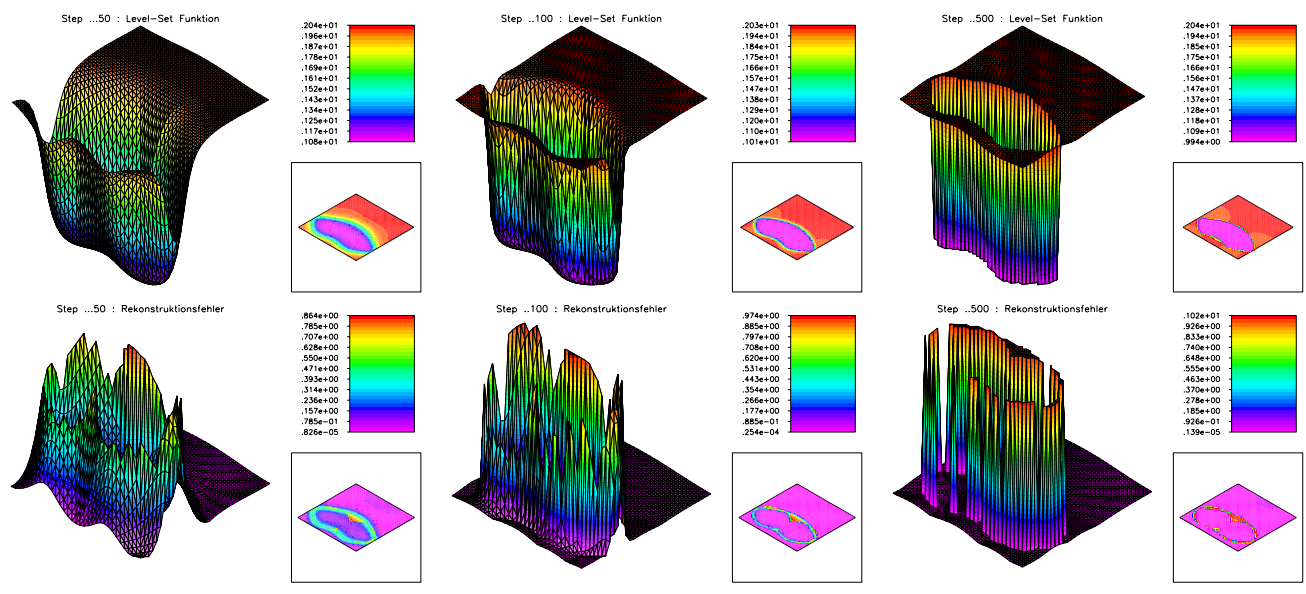

Figure 7. Third experiment: non-convex inclusion. PCLS with the Tikhonov method and non constant $\mu$. Exact data. On the first line, plots of $\phi_{k}$, for $k=50,100,500$, for the operator splitting method. The pictures on the second line show the corresponding iteration error $\left|P_{p c}\left(\phi_{k}, c^{j}\right)-u_{\text {exact }}\right|$.
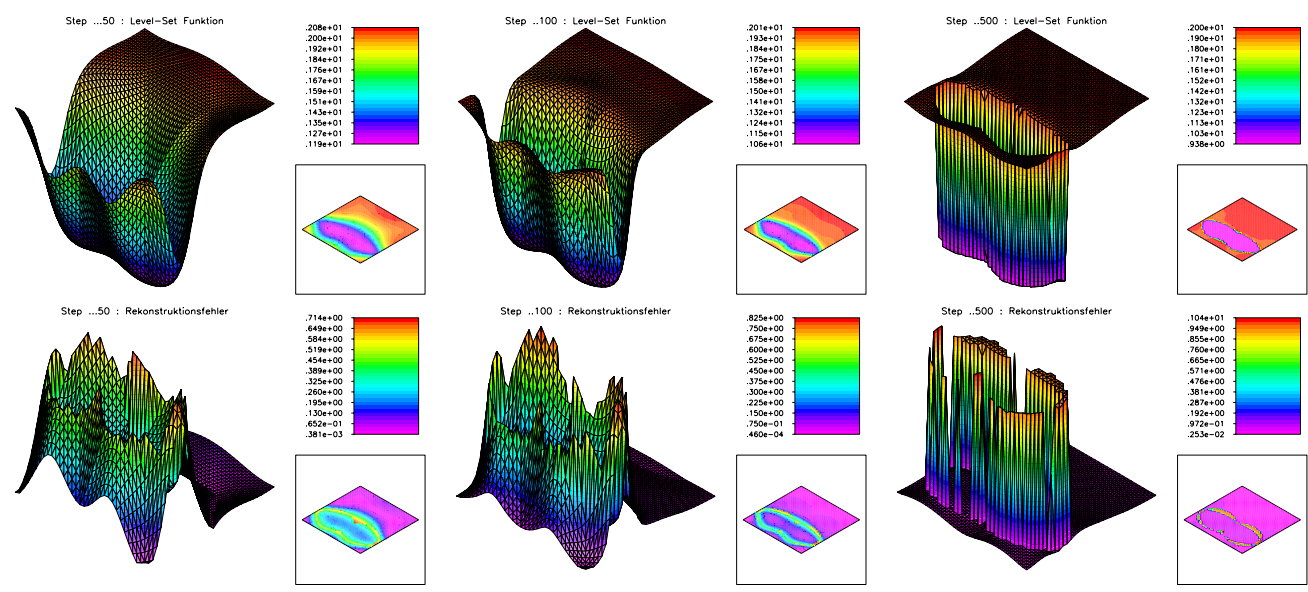

Figure 8. Third experiment: non-convex inclusion. PCLS with augmented Lagrange. Exact data. On the first line, plots of $\phi_{k}$, for $k=50,100,500$. The pictures on the second line show the corresponding iteration error.

- The first approach (Tikhonov regularization) corresponds to an extension of the results obtained in [13] for $H^{1}$ level-set functions. Convergence analysis is investigated.

- In the second approach (augmented Lagrangian), a concept of generalized multipliers (supporting an exact representation) is applied, in order to characterize the solution of a constrained optimization problem related to the original inverse problem. What concerns convergence analysis, existence of minimizers is proved (following [35, 30]), but convergence/stability results are still ongoing work.

Both solution approaches lead to iterative methods of level-set type, which are implemented and tested for a two-dimensional inverse potential problem.

To the best of our knowledge, there is no theoretical result in the literature for constrained Tikhonov regularization that could be compared to our existence result for the augmented 
Lagrangian method (theorems 9 and 11). In the literature, one can find other theoretically substantiated methods for constrained Tikhonov regularization, e.g., [3, 22, 26, 35]. However, none of these papers deal with non-convex discontinuous operator equations (which appear in our methods due to the PCSL approach). In contrast, the above-mentioned texts make use of strong regularity assumptions (smoothness and convexity) for the operator equations and for the penalization terms.

\section{Acknowledgments}

AL acknowledges support from CNPq, grant 303098/2009-0, and from the Alexander von Humboldt foundation AvH. ADC acknowledges support from PCI/MCT/IMPA 2012.

\section{References}

[1] Aubert G and Kornprobst P 2006 Mathematical Problems in Image Processing 2nd edn (Applied Mathematical Sciences vol 147) (New York: Springer)

[2] Barles G, Soner H M and Souganidis P E 1993 Front propagation and phase field theory SIAM J. Control Optim. 31 439-69

[3] Bertsekas D P 1982 Constrained Optimization and Lagrange Multiplier Methods (Computer Science and Applied Mathematics) (New York: Academic)

[4] Bourdin B and Chambolle A 2003 Design-dependent loads in topology optimization ESAIM Control Optim. Calc. Var. 9 19-48 (electronic)

[5] Burger M 2009 Finite element approximation of elliptic partial differential equations on implicit surfaces Comput. Vis. Sci. 12 87-100

[6] Burger M and Osher S 2005 A survey on level set methods for inverse problems and optimal design Eur. $J$. Appl. Math. 16 263-301

[7] Chan T F and Tai X-C 2003 Identification of discontinuous coefficients in elliptic problems using total variation regularization SIAM J. Sci. Comput. 25 881-904

[8] Chan T F and Tai X-C 2004 Level set and total variation regularization for elliptic inverse problems with discontinuous coefficients J. Comput. Phys. 193 40-66

[9] Chung E T, Chan T F and Tai X-C 2005 Electrical impedance tomography using level set representation and total variational regularization J. Comput. Phys. 205 357-72

[10] Chung J and Vese L 2003 Image segmentation using a multilayer level-sets approach UCLA C A M Rep. $1931-28$

[11] Dacorogna B 1982 Weak Continuity and Weak Lower Continuity of Nonlinear Functionals (Lecture Notes in Mathematics vol 922) (New York: Springer)

[12] De Cezaro A and Leitão A 2012 Level-set approaches of $L_{2}$-type for recovering shape and contrast in ill-posed problems Inverse Problems Sci. Eng. 20 571-87

[13] De Cezaro A, Leitão A and Tai X-C 2009 On multiple level-set regularization methods for inverse problems Inverse Problems 25035004

[14] Dorn O and Lesselier D 2006 Level set methods for inverse scattering Inverse Problems 22 R67-R131

[15] Badia A El and Farah M 2006 Identification of dipole sources in an elliptic equation from boundary measurements: application to the inverse EEG problem J. Inverse Ill-Posed Problems 14 331-53

[16] Engl H W, Hanke M and Neubauer A 1996 Regularization of Inverse Problems (Mathematics and its Applications vol 375) (Dordrecht: Kluwer)

[17] Evans L C and Gariepy R F 1992 Measure Theory and Fine Properties of Functions (Studies in Advanced Mathematics) (Boca Raton, FL: CRC Press)

[18] Frick K, Dirk A L and Resmerita E 2010 Morozov's principle for the augmented Lagrangian method applied to linear inverse problems arXiv:1010.5181v1

[19] Frick K and Scherzer O 2010 Regularization of ill-posed linear equations by the non-stationary augmented Lagrangian method J. Integral Equns Appl. 22 217-57

[20] Frühauf F, Scherzer O and Leitão A 2005 Analysis of regularization methods for the solution of ill-posed problems involving discontinuous operators SIAM J. Numer. Anal. 43 767-86

[21] Glowinski R and Le Tallec P 1989 Augmented Lagrangian and Operator-Splitting Methods in Nonlinear Mechanics (SIAM Studies in Applied Mathematics vol 9) (Philadelphia, PA: SIAM) 
[22] Hettlich F and Rundell W 1996 Iterative methods for the reconstruction of an inverse potential problem Inverse Problems 12 251-66

[23] Isakov V 1990 Inverse Source Problems (Mathematical Surveys and Monographs vol 34) (Providence, RI: American Mathematical Society)

[24] Isakov V 2006 Inverse Problems for Partial Differential Equations 2nd edn (Applied Mathematical Sciences vol 127) (New York: Springer)

[25] Ito K and Kunisch K 2008 Lagrange Multiplier Approach to Variational Problems and Applications (Advances in Design and Control vol 15) (Philadelphia, PA: SIAM)

[26] Koko J and Jehan-Besson S 2010 An augmented Lagrangian method for $t v_{g}=l^{1}$-norm minimization $J$. Math. Imaging Vis. 38 182-96

[27] Leitão A and Scherzer O 2003 On the relation between constraint regularization, level sets and shape optimization Inverse Problems 19 L1-11

[28] Litman A, Lesselier D and Santosa F 1998 Reconstruction of a two-dimensional binary obstacle by controlled evolution of a level-set Inverse Problems 14 685-706

[29] Lu T, Neittaanmäki P and Tai X-C 1991 A parallel splitting up method and its application to Navier-Stokes equations Appl. Math. Lett. 4 25-29

[30] Melo J D M 2009 On general augmented Lagrangian and a modified subgradient algorithm IMPA PhD Thesis IMPA, Rio de Janeiro

[31] Misici L and Zirilli F 1989 The inverse gravimetry problem: an application to the northern San Francisco craton granite J. Optim. Theory Appl. 63 39-49

[32] Nielsen L K, Tai X-C, Aanonsen S I and Espedal M 2007 Image segmentation by piecewise constant MumfordShah model without estimating the constants Int. J. Numer. Anal. Modelling 4 74-99

[33] Nocedal J and Wright S J 2006 Numerical Optimization 2nd edn (Springer Series in Operations Research and Financial Engineering) (New York: Springer)

[34] Rockafellar R T 1970 Convex Analysis (Princeton Mathematical Series No. 28) (Princeton, NJ: Princeton University Press)

[35] Rockafellar R T and Wets R J-B 1998 Variational analysis Grundlehren der Mathematischen Wissenschaften (Fundamental Principles of Mathematical Sciences vol 317) (Berlin: Springer)

[36] Santosa F 1996 A level-set approach for inverse problems involving obstacles ESAIM Control Optim. Calc. Var. 1 17-33

[37] Tai X-C and Chan T F 2004 A survey on multiple level set methods with applications for identifying piecewise constant functions Int. J. Numer. Anal. Modelling $125-47$

[38] Tai X-C and Li H 2007 A piecewise constant level set method for elliptic inverse problems Appl. Numer. Math. 57 686-96

[39] van den Doel K and Ascher U M 2006 On level set regularization for highly ill-posed distributed parameter estimation problems J. Comput. Phys. 216 707-23

[40] van den Doel K, Ascher U M and Leitão A 2010 Multiple level sets for piecewise constant surface reconstruction in highly ill-posed problems J. Sci. Comput. 43 44-66

[41] van den Doel Kees, Ascher Uri M and Pai Dinesh K 2008 Computed myography: three-dimensional reconstruction of motor functions from surface EMG data Inverse Problems 24065010

[42] Wu C, Zhang J and Tai X-C 2011 Augmented Lagrangian method for total variation restoration with nonquadratic fidelity Inverse Problems Imaging 5 237-61

[43] Zhou Y Y and Yang X Q 2009 Duality and penalization in optimization via an augmented Lagrangian function with applications J. Optim. Theory Appl. 140 171-88

[44] Zhu S and Liu C 2004 A semi-implicit binary level set method for source reconstruction problems Int. J. Numer. Anal. Modelling 1 1-18 